\title{
Nanomedicine-based technologies and novel biomarkers for the diagnosis and treatment of Alzheimer's disease: from current to future challenges
}

Amanda Cano ${ }^{1,2,3,4^{*}}$, Patric Turowski ${ }^{5}$, Miren Ettcheto ${ }^{2,6}$, Jason Thomas Duskey ${ }^{7,8}$, Giovanni Tosi ${ }^{7}$, Elena Sánchez-López ${ }^{2,3,4}$, Maria Luisa García ${ }^{2,3,4}$, Antonio Camins ${ }^{2,6}$, Eliana B. Souto 9,10, Agustín Ruiz 1,2, Marta Marquié ${ }^{1,2 \dagger}$ and Mercè Boada ${ }^{1,2 \dagger}$

\begin{abstract}
Increasing life expectancy has led to an aging population, which has consequently increased the prevalence of dementia. Alzheimer's disease (AD), the most common form of dementia worldwide, is estimated to make up 50-80\% of all cases. AD cases are expected to reach 131 million by 2050, and this increasing prevalence will critically burden economies and health systems in the next decades. There is currently no treatment that can stop or reverse disease progression. In addition, the late diagnosis of AD constitutes a major obstacle to effective disease management. Therefore, improved diagnostic tools and new treatments for AD are urgently needed. In this review, we investigate and describe both well-established and recently discovered AD biomarkers that could potentially be used to detect AD at early stages and allow the monitoring of disease progression. Proteins such as NfL, MMPs, p-tau217, YKL-40, SNAP-25, VCAM-1, and Ng / BACE are some of the most promising biomarkers because of their successful use as diagnostic tools. In addition, we explore the most recent molecular strategies for an AD therapeutic approach and nanomedicine-based technologies, used to both target drugs to the brain and serve as devices for tracking disease progression diagnostic biomarkers. State-of-the-art nanoparticles, such as polymeric, lipid, and metal-based, are being widely investigated for their potential to improve the effectiveness of both conventional drugs and novel compounds for treating AD. The most recent studies on these nanodevices are deeply explained and discussed in this review.
\end{abstract}

Keywords: Alzheimer's disease, Biomarkers, Polymeric nanoparticles, Lipid nanoparticles, Metal nanoparticles, Nanotechnology

*Correspondence: acano@fundacioace.org; acanofernandez@ub.edu ${ }^{\dagger}$ Marta Marquié and Mercè Boada-senior co-authors

${ }^{1}$ Research Center and Memory Clinic, Fundació ACE. Institut Català de Neurociències Aplicades, International University of Catalunya (UIC), C/ Marquès de Sentmenat, 57, 08029 Barcelona, Spain

Full list of author information is available at the end of the article

\section{Highlights}

- Alzheimer's disease is the most common form of dementia worldwide, estimated to be responsible for $50-80 \%$ of all cases.

- No currently available treatment can stop the disease progression, and it is expected that $\mathrm{AD}$ cases will reach 131 million by 2050 .

- The late diagnosis of AD will have a serious impact on health and socio-economic systems worldwide.

(c) The Author(s) 2021. This article is licensed under a Creative Commons Attribution 4.0 International License, which permits use, sharing, adaptation, distribution and reproduction in any medium or format, as long as you give appropriate credit to the original author(s) and the source, provide a link to the Creative Commons licence, and indicate if changes were made. The images or other third party material in this article are included in the article's Creative Commons licence, unless indicated otherwise in a credit line to the material. If material is not included in the article's Creative Commons licence and your intended use is not permitted by statutory regulation or exceeds the permitted use, you will need to obtain permission directly from the copyright holder. To view a copy of this licence, visit http://creativeco mmons.org/licenses/by/4.0/. The Creative Commons Public Domain Dedication waiver (http://creativecommons.org/publicdomain/ zero/1.0/) applies to the data made available in this article, unless otherwise stated in a credit line to the data. 
- Novel biomarkers, such as NfL, SNAP-25, Clusterin, MMPs, and YKL-40, among others, are being studied alongside commonly used biomarkers as potential new diagnostic tools.

- Nanomedicine has aroused much interest in the last decade and has shown promising results for improving drug targeting and delivery to the brain.

- Polymeric-, lipid-, and metal-based nanoparticles are the most commonly investigated nanodevices in the field of dementia.

- State-of-the-art nanoparticles are emerging as promising tools to improve the diagnosis and treatment of AD.

\section{Introduction}

In the past few decades, the number of people living with dementia has increased exponentially all around the world, mainly due to aging populations and improvements in quality of life. The latest Global Burden Disease Study estimated that the number of cases of dementia more than doubled from 1990 to 2016 and indicated population growth and aging as the main drivers of this increase (Fig. 1) [1].
Alzheimer's disease (AD) is the most common form of dementia worldwide, estimated to constitute up to $50-80 \%$ of cases [1]. Undoubtedly, AD will critically burden economies and health systems since cases are expected to reach 131 million by 2050 [2]. AD is commonly diagnosed symptomatically through the occurrence of significant memory loss, global cognitive decline, and the impairment of daily life activities. Later in the course of the disease, the breakdown of physical functions, such as walking, swallowing, and general movement, ultimately leads to death [3]. Dementia was the fifth-leading cause of death in 2016 [1].

$\mathrm{AD}$ can be classified depending on the onset of the first symptoms. Approximately $1-6 \%$ of all cases are categorized as early-onset $\mathrm{AD}$ (EOAD), which manifests before the age of 65 . By contrast, late-onset AD (LOAD) is characterized by the occurrence of symptoms at an age greater than 65 years and accounts for around 90\% of cases [4]. EOAD differs from LOAD in many aspects. Prominently, EOAD has a more aggressive course of disease progression, greater delay to diagnosis, lower cognitive reserves, lower incidence of diabetes, obesity, and circulatory disorders, relatively greater deficits in attention, executive functions, praxis, and visuospatial

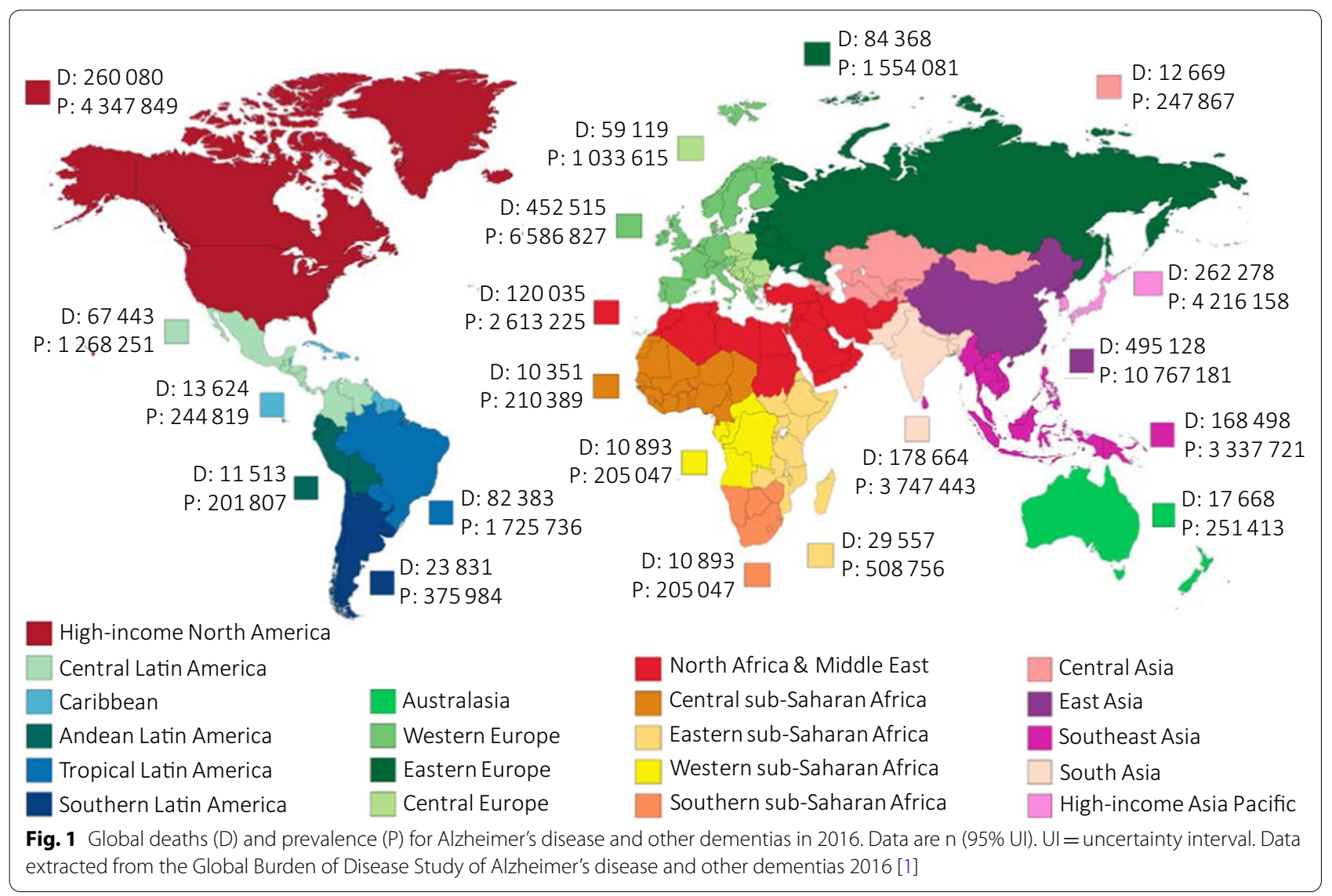


functions, lower frequency of the APOE $\varepsilon 4$ allele, greater white matter changes, and a higher burden of neuritic plaques and neurofibrillary tangles [5].

In general, $\mathrm{AD}$ is defined as a multifactorial disease in which genetic, environmental, behavioral, and developmental components critically influence its pathogenesis, with age being the most important risk factor. Although most cases of $\mathrm{AD}$ are sporadic, rare cases $(<1 \%)$ have a genetic component ("Familial AD", FAD) with a few hundred families identified worldwide. These cases usually have an early onset at young ages (40-50). The three known genes that cause FAD, with an autosomal dominant inheritance, are the amyloid precursor protein (APP), presenilin-1 (PS1), and presenilin-2 (PS2) genes, all of which are involved in the processing or production of $A \beta$ [6]. Our understanding of the pathophysiology of $\mathrm{AD}$ is constantly changing [4]. The overproduction and accumulation of amyloid- $\beta(A \beta)$ peptide and hyperphosphorylated tau (p-tau) protein are the two most common hypotheses for $\mathrm{AD}$ pathogenesis, but recent findings have demonstrated that chronic oxidative stress, hormone imbalance, mitochondrial dysfunction, inflammation, calcium mishandling, mitotic dysfunction, genetic components or blood-brain barrier (BBB) dysfunction also likely play key roles in the disease process $[7,8]$. Similarly, emerging studies have also reported that oligodendrocytes act as antigen-presenting cells and produce immune molecules. Likewise, the activation of both astroglia and oligodendrocytes, mainly due to $\mathrm{BBB}$ dysfunction and general toxic species produced in $A D$, occurs widely in $\mathrm{AD}$ [9]. In neuroinflammation processes, oligodendrocytes express many factors known to activate astrocytes. In response to these factors, astrocytes and oligodendrocytes secrete immune factors, underscoring the possible immune function of these cells. IL-1 $\beta$, IL17 tumor necrosis factor- $\alpha$ (TNF- $\alpha$ ), interferon- $\gamma$ (IFN- $\gamma$ ), and fibroblast growth factor-2 (FGF-2) are some of these secreted cytokines that induce pro-inflammatory effects [9]. Regardless of all these changes, neuronal death is the ultimate invariable outcome, which then drives the typical neurodegeneration of AD.

Currently there are only four FDA approved treatments for $\mathrm{AD}$, and these are linked mainly to the two molecular pathways involving the accumulation of $A \beta$ peptide and neurofibrillary tangles (NFT) of p-tau protein [10]. However, none of these drugs stops disease progression or cures $\mathrm{AD}$, highlighting the need for additional treatment approaches. The discovery of novel biomarkers is hoped to deliver earlier AD diagnosis and could also support the identification of additional molecular targets, potentially leading to new treatments.

Identifying the pathophysiological processes involved in $\mathrm{AD}$ and the best biomarkers to detect them is critical for the development of novel cures. In addition, efficiently and specifically delivering the diagnostic and therapeutic molecules to the sites of interest in these processes is critical. Nanoparticles (NPs) have enabled great strides towards the delivery, treatment and diagnostics of diseases, mainly due to their various chemical characteristics and their propensity for chemical modification to modulate and refine required properties [11, 12]. NPs' core constituents comprise a wide variety of materials, such as lipids, polymers, and metals, that can encapsulate molecules with different chemical natures. In addition, these carriers promote the protection and delivery of bioactive molecules, which can reduce their potential toxicity and, in turn, enhance their solubility, stability, biodistribution, and pharmacokinetics. Molecules encapsulated in NPs range from small molecules, peptides, and proteins to genetic material [13]. Crucially for chronic CNS diseases, NPs have the demonstrated capacity to deliver molecules to hard-to-reach tissues, such as the CNS where the crossing of the BBB and the release of drugs with controlled kinetics for long-term treatments are required [11, 14].

In this review, we investigate and describe well-established AD biomarkers and highlight recently discovered ones that could be potential diagnosis tools. In addition, we explore the most recent nanomedicine-based technologies used to target drugs across the $\mathrm{BBB}$ and increase CNS delivery of these active molecules.

\section{Novel biomarkers in the diagnosis of Alzheimer's Disease}

AD was first described in 1906 [15]. For a long time after that, firm diagnosis was made when signs of memory loss and cognitive decline were already significantly advanced. Nowadays, many observations indicate that the pathophysiological alterations of AD in the brain begin decades before the onset of clinical symptoms of dementia. Historically, AD patients were classified into three clinical categories: cognitively unimpaired $(\mathrm{CU})$, mild cognitive impairment (MCI), and dementia patients [16]. AD is now recognized as a continuum of neurological decline that can be identified and staged through a combination of neuropathological findings and in vivo biomarkers $[17,18]$. This has led to a paradigm shift in the diagnosis of $\mathrm{AD}$, opening new windows of opportunities for early treatment in the preclinical stages. In that context, the National Institute on Aging and the Alzheimer's Association have recently updated diagnostic criteria, with a clear shift from a clinical to a biological definition of $\mathrm{AD}$ [19].

Biomarkers now have key importance in the robust diagnosis of $\mathrm{AD}$. Biomarkers are quantifiable molecules or processes that can be related to the biological 
alterations and/or pharmacologic responses to a therapeutic intervention for a specific disease $[18,20]$. An ideal biomarker should be specific, sensitive, predictive, accurate, robust, inexpensive, and ideally non-invasive and measurable in common biological fluids such as serum, saliva and/or urine [21, 22]. For AD, cerebrospinal fluid (CSF) is considered the optimal biological source for biomarker assessment, since its direct contact with the interstitial fluid where the brain is immersed reflects the pathophysiological changes of AD progression in real time $[18,23]$. For AD biomarkers, specificity and sensitivity of more than $80 \%$ are needed to be considered a reliable biomarker [18]. Notably, not all patients with cognitive impairment develop $A \beta$ plaques or tau neurofibrillary tangles (NFTs), and vice versa. Furthermore, biomarkers have different uses and interpretations depending on the degree of the cognitive deterioration observed. Thus, the use of biomarkers has allowed the identification of prodromal AD in MCI subjects and the confirmation of AD in individuals with dementia [18].

Several biomarkers are currently being used in a complementary way with conventional neuropsychological tests and routine neurological exams in the diagnosis of AD. The parameters and diagnostic implications of these conventional biomarkers have recently been reviewed and updated. Furthermore, new molecules, related to different pathophysiological pathways involved in the development of $\mathrm{AD}$, are being identified as potential new biomarkers for the early diagnosis of this disorder (Fig. 2) [24-27].

\section{Conventional AD biomarkers in CSF/plasma $A \beta$-related biomarkers}

In $A D$ pathogenesis, the production of $A \beta$ peptides is increased, while their clearance is reduced. $A \beta 1-40$ and $A \beta 1-42$ fragments predominantly form, promoting the aggregation of these peptides and thus leading to the formation of senile plaques [28]. A $\beta 42$ is one of the most toxic isoforms of the $A \beta$ peptide and is also one of the most widely accepted biomarkers of AD diagnosis [29, 30]. $A \beta 42$ quantification in CSF allows the identification of $\mathrm{AD}$ in its preclinical stage, and possesses high diagnostic value with clear specificity for $\mathrm{AD}$ over other neurodegenerative diseases. However, absolute $\mathrm{A} \beta 42$ levels vary due to a variety of factors, particularly interindividual differences [29]. To account for these, the ratio between $A \beta 42 / A \beta 40$ is also commonly used. $A \beta 40$ concentration is 10-times higher than $A \beta 42$ in CSF and its level does not usually vary in AD [31]. Similarly, the $A \beta 42 / A \beta 38$ ratio is emerging as a potential predictive tool with comparable power to the $A \beta 42 / A \beta 40$ ratio [32-34].

Likewise, as with other disease biomarkers, current efforts are also focused on improving $A \beta 42$ detection in blood samples $[28,29]$. CSF extraction through lumbar

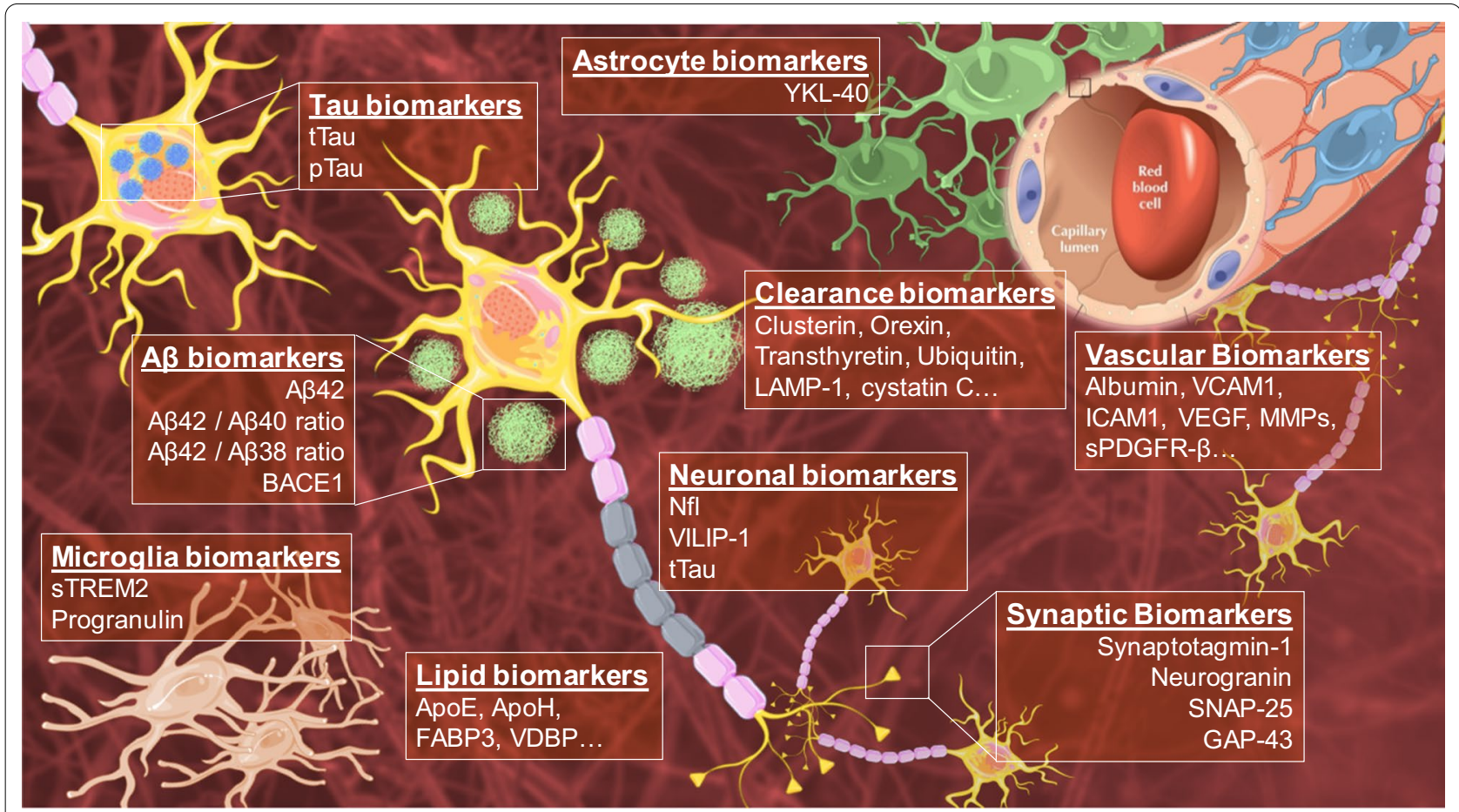

Fig. 2 Conventional and novel biomarkers related to molecular alterations and physiopathological changes of AD 
function is an invasive procedure that is only permitted for certain patients. It is often not feasible, e.g., for patients with low back injuries, increased intracranial pressure due to a space-occupying lesion, coagulopathy, or infected skin over the puncture site [35, 36]. Immunoprecipitation coupled with mass spectrometry, ELISA, and protein misfolding cyclic amplification assays are possible techniques for detecting blood $\mathrm{A} \beta 42[29,37]$. Recently, a blood analytical test for the detection of plasma $A \beta$ by mass-spectrometry gained approval by regulators at the Centers for Medicare \& Medicaid Services under the Clinical Laboratory Improvement Amendments protocol [38]. Researchers predict that the next tests to be approved are plasma p-tau181 and p-tau217, as these tests will be more robust since they possess a larger dynamic range. Indeed, phosphorylated fragments of tau increase by as much as tenfold in the blood as AD progresses, whereas the $A \beta 42 / 40$ ratio falls by about 15 percent at most [38].

BACE1, the key enzyme that initiates the formation of $A \beta$ peptide [39], has increasingly been studied as a potential AD biomarker. BACE1 can be measured in CSF, but there is controversy regarding its predictive value. Some studies have shown increased levels and protein activity of BACE1 in AD patients and that this is a good predictor of the progression of MCI patients [40, 41]. However, more recent studies have reported no changes in CSF BACE1 levels among controls, MCI and AD patients [42-44].

\section{Tau-related biomarkers}

It is widely accepted that hyperphosphorylated tau (p-tau), accumulating as intracellular NFTs, is the other main pathological hallmark of $\mathrm{AD}$, together with $\mathrm{A} \beta$ plaques $[29,45]$. However, in many neurodegenerative diseases, collectively known as "tauopathies", p-tau NFTs are found that differ in their phosphorylated residues $[29,46]$. The diagnostic value of total tau (t-tau) levels for differentiating AD from normal aging is well described. $\mathrm{T}$-tau has been considered a relevant biomarker of neuronal injury and thus is not a specific marker of $\mathrm{AD}$; it is also found to be elevated in other neurological conditions such as Creutzfeldt-Jakob's disease, frontotemporal dementia with parkinsonism, and Pick's disease, among others [47]. P-tau (specifically tau phosphorylated on threonine 181, p-tau181) has been described as a more specific biomarker for $\mathrm{AD}$ since $\mathrm{p}$-tau is present at normal levels in most other neurodegenerative disorders, but not in AD [29]. In clinical practice, the correlation between both $\mathrm{t}$-tau and p-tau in CSF is important for differentiating among several dementia types [48].

Levels of tau deposition display a stronger correlation to cognitive decline than does $\mathrm{A} \beta$ in $\mathrm{AD}$ patients [49].
However, longitudinal studies have revealed that CSF tau levels vary depending on the different stages of the disease $[49,50]$. Several recent studies have tried to elucidate the prognostic value of tau in early-onset $\mathrm{AD}$ and as a biomarker to monitor drug responses, but further investigation is required [29].

As with $A \beta$, progress is also being made regarding the predictive value of tau in blood samples thanks to ultrasensitive immunoassays techniques. Some studies have already identified higher plasma and/or serum p-tau181 levels in $\mathrm{AD}$ patients versus controls using innovative techniques such as Simoa ${ }^{\circledR}$, immunomagnetic reduction, and label-free real-time surface plasmon resonance [51-53]. Interestingly, recent findings have shown the predictive value of plasma $\mathrm{p}$-tau217 for discriminating $\mathrm{AD}$ from other neurodegenerative diseases, specifically as an accurate predictor of $\beta$-amyloidosis at asymptomatic and symptomatic stages [54-57]. In these studies, p-tau-217 and p-tau-181 were highly specific for amyloid plaque pathology, as p-tau-217 measurement was still more specific to amyloid status than p-tau-181 [54-57]. Collectively, these findings raise the possibility for a novel, highly sensitive, and specific biomarker based on circulating tau, with improved diagnostic accuracy. Further studies are needed to validate these findings in unselected and diverse populations and determine its potential role in clinical practice [54-57].

\section{Novel AD biomarkers in CSF/plasma Neuronal damage-related biomarkers}

Other molecules and cellular structures have been suggested as potential biomarkers of neuronal damage. Among these, neurofilaments, specifically neurofilament light chain (NfL), have emerged as promising biomarkers. After axonal and/or neuronal damage, NfL leaks into CSF and increases to detectable levels [58].

Although NfL is not specific to AD, it has been shown to be intimately linked to neurodegenerative diseases, and its predictive value increases in combination with other biomarkers [59]. However, one of the benefits of NfL compared to other biomarkers is the high correlation of its levels in CSF and blood [60]. Moreover, similarly to $A \beta$, increased levels of NfL can be detected in the early stages of autosomal dominant $\mathrm{AD}$, even before the onset of first symptoms [61]. In addition, a recent 18-month trial with mild AD patients showed that NfL, as a Tau-independent marker of axonal degeneration, had a stronger association with clinical scales than did $\mathrm{t}$-tau [62]. Thus, the ability of NfL to detect changes before clinical manifestations occur reveals its promise as a diagnostic biomarker.

Visinin-like protein 1 (VILIP-1), a calcium sensor protein highly expressed in neurons, is another protein that 
could act as a biomarker of neuronal-injury in AD [18, 29]. This is supported by the strong correlation between increased levels of both VILIP-1 and tau in the CSF of $A D$ patients [63, 64]. Importantly, some studies have reported the predictive value of VILIP-1 for atrophy rates and cognitive decline, indicating that this protein could help identify MCI patients that will progress to $\mathrm{AD}$ [64]. In blood samples however, the predictive value of VILIP-1 remains uncertain [27, 29].

\section{Neuroinflammation-related biomarkers}

Inflammatory processes play important roles in the pathogenesis of AD. The activation of glial cells, the resident immune cells of the CNS, is well described for different neurodegenerative diseases, especially $\operatorname{AD}[65,66]$. Historically, it was accepted that neuroinflammation, initiated primarily as a reaction to $A \beta$ and $p$-tau neurotoxicity, triggered the production of neurotoxic molecules (e.g. ROS, glutamate or inflammatory cytokines). This pattern is reproduced in a continuous molecular feedback loop, which has been described to be more pronounced in late stages of $\mathrm{AD}$ [67]. However, recent evidence also reveals the neuroprotective role of microglia and astroglia in earlier stages of AD development [67, 68]. When the deposition of $A \beta$ plaques appears, glial cells create an immune barrier that surrounds and isolates these senile plaques. This process protects the axons adjacent to $A \beta$ plaques from $A \beta$ neurotoxicity $[50,60]$. Recent genetic studies have found correlations between the deficiency of microglia encapsulation toward $A \beta$ deposits and an increased risk of late-onset $\mathrm{AD}[53,58]$. In addition, an inverse correlation between neuroinflammation in the brain cortex and plasmatic NfL levels has been reported $[69,70]$. In normal aging, serum levels of NfL show a nonlinear increase from 60 years old in both males and females [71]. However, low plasma NfL levels are associated with raised cortical microglial activation, suggesting that inflammation acts to protect prodromal AD [70].

Consequently, neuroinflammation biomarkers may be of great interest for the early diagnosis of preclinical AD. YKL-40, also known as human cartilage glycoprotein 39 and predominantly related to astroglial proteins, is one of the most studied neuroinflammation-related biomarkers [72]. YKL-40 is not only upregulated in $\mathrm{AD}$, but in other diseases in which inflammation plays an important role [72]. Longitudinal studies and recent meta-analyses have reported that CSF YKL-40 levels are higher in AD patients compared with controls, increase throughout the disease progression and are positively correlated with neuronal-injury biomarkers, mainly in the preclinical stages of AD [30, 64]. However, YKL-40 has not yet shown consistent results in blood samples.
Progranulin, a glycoprotein mainly secreted by activated microglia, is involved in the modulation of neuroinflammation [73]. This protein has aroused much interest since its loss-of-function has been closely correlated with some types of frontotemporal lobar degeneration [29, 74]. However, although a cross-sectional study reported increased CSF progranulin levels in autosomal-dominant $\mathrm{AD}$ and late-onset $\mathrm{AD}$, progranulin from CSF or blood is currently not validated as diagnostic markers $[75,76]$.

Another neuroinflammation-related biomarker that has attracted attention is the triggering receptor expressed on myeloid cells 2 (TREM2), which has an arguably controversial role in $\mathrm{AD}$ development. This transmembrane protein belongs to the immunoglobulin family and is expressed in microglial cells [77]. TREM2 is involved in many biological processes, such as migration, proliferation, cytokine release, APOE binding, or sealing of $A \beta$ plaques $[29,68]$. Moreover, a rare missense mutation, predicted to lead to an $\mathrm{R} 47 \mathrm{H}$ substitution in TREM2, has been linked to a significant risk of $A D$ in Iceland [78]. It was also reported that the soluble ectodomain of TREM2 (sTREM2) is released into the extracellular space and may serve as a CSF biomarker [29]. However, to the best of our knowledge, the biological role of sTREM2 is unclear, particularly in neuroinflammation. Some studies have shown that hyperstimulation of the TREM2 pathway correlates to the attenuation of microglial activation [79-81]. However, further studies are needed to provide firm mechanistic evidence.

Taken together, most of these inflammation-related biomarkers are not specific to $\mathrm{AD}$ and have thus been proposed as "neuroinflammation-tracking assistants" that can provide a more solid diagnosis together with specific AD biomarkers. Additionally, they could be used to identify AD patients who would benefit from novel microglia-targeted treatments.

\section{Synaptic dysfunction-related biomarkers}

Synaptic degeneration is another hallmark of early $\mathrm{AD}$ pathology and appears to closely correlate with cognitive decline [82]. This event is associated, in turn, with the neurotoxic effects of $A \beta$ and tau species and glutamatergic excitotoxicity, which lead to alterations in axonal transport that later promote dendritic alterations and eventually neuronal loss [83]. Many molecules involved in synaptic degeneration have been identified as potential biomarkers and have been divided into pre- and postsynaptic biomarkers.

Growth-associated protein 43 (GAP-43) belongs to the group of potential presynaptic biomarkers [27, 29]. This protein is involved in synaptogenesis and neuronal development and is mainly expressed in the cortex, hippocampus, olfactory bulb, and cerebellum. In fact, three 
decades ago, the correlation between an increased density of neurons containing NFTs and decreased levels of GAP-43 in the brain and cerebellar cortex of AD patients had already been reported [84]. More recently, the significant elevation of CSF GAP-43 in AD patients has been reported [85]. Interestingly, this increase also correlates with the amount of $A \beta$ and p-tau found in the cortex, amygdala and hippocampal structures [85]. These findings indicate that GAP-43 is a promising biomarker for AD in CSF. However, its usefulness in blood samples has not yet been well described.

Synaptosomal-associated-protein-25 (SNAP-25) is a newly discovered potential biomarker related to pre-synaptic damage. Recent studies reported that CSF SNAP-25 increases in $\mathrm{MCI}$ and $\mathrm{AD}$, which is associated with the rate of hippocampal atrophy and cognitive decline [86]. Moreover, CSF SNAP-25 levels are also substantially higher in MCI patients who are APOE $\varepsilon 4$ carriers, compared to non-carriers. These findings suggest the potential relevance of SNAP-25 to predict the conversion from $\mathrm{MCI}$ to AD [87]. SNAP-25 may even have relevance as a blood biomarker. Agliardi et al. showed that SNAP-25 can be detected in serum, where it appears in neuronderived plasma exosomes, which are extracellular vesicles involved in intercellular communication. Serum levels of SNAP-25 are lower in AD patients compared to healthy controls [88]. Although further studies are needed, these findings indicate the potential use of SNAP- 25 as a presynaptic injury-related biomarker.

Synaptotagmin-1 was one of the first proteins detected in the CSF of early-onset AD patients [89]. Synaptotagmin-1 is a pre-synaptic vesicle protein involved in the maintenance of correct synaptic transmission and cognitive function. Recent studies reported increased CSF Synaptotagmin-1 levels in both MCI and AD patients compared to healthy controls [90], indicating that Synaptotagmin-1 is a potential CSF biomarker of both $\mathrm{AD}$ and conversion from $\mathrm{MCI}$ to $\mathrm{AD}$.

Neurogranin $(\mathrm{Ng})$ is a post-synaptic protein that regulates calcium signaling and synaptic plasticity mainly found in the dendritic spines of the amygdala, hippocampus, and caudoputamen $[29,82]$. High CSF Ng levels have been reported to predict future cognitive decline specific to AD pathogenesis, with better specificity than p-tau [91]. Furthermore, several studies have shown elevated CSF Ng levels in MCI and prodromal AD patients [91, 92]. Interestingly, a recent study carried out by Kirsebom et al. identified an elevated CSF Ng/BACE1 ratio as a predictor of very early cognitive decline in AD. Specifically, elevated $\mathrm{Ng} / \mathrm{BACE} 1$ is associated with lower hippocampal and amygdala volumes of MCI patients compared to healthy controls [93]. All these findings highlight $\mathrm{Ng}$ as one of the most relevant biomarkers related for synaptic dysfunction in AD. The scientific evidence to date has not clarified the predictive value of blood $\mathrm{Ng}$ as an $\mathrm{AD}$ synaptic-damage biomarker. In addition, further studies are needed to define the specific range of $\mathrm{Ng}$ values for diagnoses of different stages of AD.

\section{BBB dysfunction-related biomarkers}

Cerebrovascular disease and AD share multiple risk factors. Substantial evidence suggests that vascular dysfunction is the earliest event in the pathogenic development of late-onset AD [94]. Vascular dysfunction, associated with aging, causes reduced oxygen, glucose, and nutrient supply to the brain, which directly damages not only the parenchymal cells, but also the BBB structure. This in turn promotes the overproduction of ROS, nitric oxide, and inflammatory cytokines in response to the neurotoxic effects of vascular dysfunction, which contributes to a vicious circle of global neurotoxicity affecting both $\mathrm{BBB}$ dysfunction and AD pathogenesis [94]. The standard biomarker of BBB dysfunction measured in clinical practice is the CSF/serum ratio of albumin [95]. Although the role of albumin and its predictive value are still controversial, many efforts have been made to exploit the usefulness of this biomarker. Other molecules have also emerged as potential biomarkers of BBB-dysfunction, such as VCAM-1, ICAM-1, MMPs, VEGF, PIGF, sPDGFR- $\beta$, and tight junction proteins (such as claudins and occludin) $[25,29]$. However, further studies are needed to determine their predictive value in CSF, as well as their correlation in peripheral blood.

\section{Lipid metabolism-related biomarkers}

Brain tissue is highly enriched in lipids. Lipid metabolism is decisive for the synaptic activity, neuronal survival, and immune responses of glial cells, and AD pathogenesis is accompanied by continuous changes in brain lipid patterns [96]. Thus, proteins related to lipid transport and metabolism in the CNS have been proposed as potential biomarkers of AD pathogenesis. Undoubtedly, ApoE is the prototypical protein involved in lipid homeostasis and $\mathrm{AD}$ development. The expression of the ApoE $\varepsilon 4$ allele is well known as the strongest genetic risk factor for AD [27, 97]. Therefore, CSF ApoE levels have been investigated as $\mathrm{AD}$ progression biomarkers and for the differential diagnosis of $\mathrm{AD}$ and other neurodegenerative disorders [27]. However, further studies are needed to validate its potential predictive value in blood samples. Similarly, heart fatty acid-binding protein (FABP3), $\mathrm{ApoH}$, and vitamin D-binding protein are related to lipid metabolism and increased levels of them have been found in the CSF of AD patients. Moreover, these have been proposed for the differential diagnosis of $\mathrm{AD}$ from 
Lewy body dementia, Parkinson's disease, and other dementias [27].

\section{Neurotoxins clearance-related biomarkers}

The clearance of toxic metabolites from the brain is essential for healthy function. Since the dysregulation of clearance mechanisms has been identified as a direct cause of $\mathrm{AD}$ development, proteins related to the removal of cerebral metabolic waste, $A \beta$ and $p$-tau peptides, and reactive oxidative species have been suggested as candidate biomarkers [27, 98]. Clusterin, Orexin and Transthyretin, which are involved in the clearance of partially unfolded proteins and $A \beta$ peptides, have thus been described as potential biomarkers [27]. Interestingly, clusterin, also called APOJ, is also related to lipid transport, inflammation, and chaperone activities. Similarly, LAMP-1, carboxypeptidase E, cystatin $\mathrm{C}$, and ubiquitin CSF levels are increased in patients with $\mathrm{AD}$ and are emerging as potential neurotoxin clearance-related biomarkers $[27,99]$.

\section{Metal ion homeostasis-related biomarkers}

Metal ions have been widely described as potential targets for the diagnosis and treatment of $\mathrm{AD}$. The abnormal accumulation of metal ions, like zinc, copper, and iron, in the brain has been closely related to the overproduction of $\mathrm{A} \beta$ peptide and $\mathrm{p}$-tau, and the accumulation of senile plaques and NFTs [100]. Likewise, abnormalities in metal-binding proteins are a key factor in promoting the erroneous distribution and deposition of metal ions in the brain [100]. Some of the mechanisms by which metal ions promote these abnormalities are the induction of oxidative stress, autophagy dysfunctions, the disruption of endoplasmic reticulum and mitochondria structures, activation of $\beta$ - or $\gamma$-secretases, inhibition of $\alpha$-secretase, and activation of protein kinases such as cyclindependent protein kinase-5 (CDK5), glycogen synthase

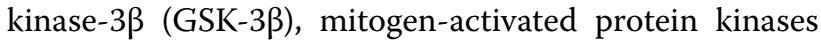
(MAPKs), etc. [100]. Moreover, all these alterations enhance the abnormal brain deposition of metal ions and further dysregulate their homeostasis. Therefore, these metal ions have been proposed as potential biomarkers for tracking $\mathrm{AD}$ progression. Likewise, adjusting metal balance may be a potential treatment for AD pathologies and is a promising path for future research.

\section{Molecular strategies for the therapeutic approach to Alzheimer's disease \\ Amyloid- $\beta$ strategies}

As explained above, the overproduction and accumulation of $A \beta$ lead to several neuropathological processes that trigger neuronal death, which translates into the memory loss and cognitive disorders typical of this disease $[28,101] . \alpha$-secretase is the main enzyme in amyloid-precursor protein (APP) metabolism and its action is followed by that of $\gamma$-secretase in physiological conditions. Amyloidogenic processing occurs when an alternate enzyme, $\beta$-secretase, acts instead of $\alpha$-secretase [102]. Generally, $A \beta$-based therapies target several aspects of APP metabolism [101]. Likewise, A $\beta$-binding to several receptors has been related with some of its neurotoxic mechanisms (Table 1) [103]. Thus, therapeutic targeting to these proteins has been proposed to decrease amyloidogenic APP processing and A $\beta$-related toxicity.

In this context, recent pre-clinical studies have suggested that epigallocatechin-gallate (EGCG), the most abundant polyphenol in green tea, induces $\alpha$-secretase activity and improves non-amyloidogenic APP processing [104]. Likewise, the effect of a one-year treatment with EGCG on cognitive A $\beta$ biomarkers, as well as metabolomics, microbiota, saliva, plasma, and urine, is being evaluated in a randomized, double-blind clinical trial in 200 subjects (NCT03978052) This study aims to demonstrate the potential prevention of cognitive decline in patients with positive ApoE\&4 with subjective cognitive decline after a multimodal intervention with EGCG [105]. The estimated study completion date is September 2021, when the first results will reveal the potential of this drug for AD.

Etazolate is another compound that has been shown to possess neuroprotective effects in $\mathrm{AD}$. It is a pyrazolopyridine with anxiolytic-like properties that selectively modulates the $\mathrm{GABA}_{\mathrm{A}}$ receptor. Marcade et al. demonstrated that Etazolate also promotes neuroprotection via the $\alpha$-secretase pathway, leading to an induction of sAPP $\alpha$, which is a neurotrophic and precognitive molecule [106]. Thus, modulators of the $\mathrm{GABA}_{\mathrm{A}}$ receptors could offer a promising opportunity for the treatment of $\mathrm{AD}$.

The active enantiomer of phenserine, which directly decreases levels of APP by interacting with APP mRNA, has also been found to be effective in enhancing $\alpha$-secretase and AChE activity [4]. However, other secretase-targeting drugs have not succeeded. For instance, tarenflurbil, the active enantiomer of flurbiprofen, ELND006 and Semagacestat are all $\gamma$-secretase inhibitors developed to reduce $A \beta$ levels that failed in clinical trials because of their significant adverse effects, and the absence of results extrapolatable from pre-clinical models to patients [4].

Modulating $\mathrm{A} \beta$ transport is another therapeutic approach to $\mathrm{AD}$. Apolipoproteins have an important role in $A \beta$ transport and metabolism since they regulate the movement of $A \beta$ peptides between the periphery and CNS. ApoE\&4 increases the passage of $A \beta$ from blood to the brain through the low-density lipoprotein 
Table 1 Main receptors and their implications in A $\beta$ binding-mediated neurotoxic effects [103]

\begin{tabular}{ll}
\hline Receptor & Localization \\
\hline NMDAR & Postsynaptically located on dendrites and dendritic spin \\
AMPAR $\quad$ Hippocampal pyramidal neurons and dendritic spines \\
PrPC & Brain neurons and spinal cord
\end{tabular}

mGluR5 Hypothalamus and cortex

B2ARs Locus coeruleus, hippocampus and cortex

a7nAChR Septo-hippocampal region and cortical neurons

IR Choroid plexus, olfactory bulb and regions of the striatum and cerebral cortex

p75NTR White matter brain regions and spinal cord

ILR

Hippocampus and surface of B lymphocytes, dendritic cells, natural killer cells, macrophages, granulocytes, mast cells, etc

TREM2 Surface of immune cells of myeloid origin

Eph4A Hippocampal neurons

EphB2

RAGE Blood-brain barrier

LPR2 Choroid plexus epithelium and ependymal cells covering the brain ventricles

VDR Broadly expressed in all brain regions

\section{Proposed mechanisms}

Impairment of NMDAR activity: removal from the cell surface and triggering of synaptic depression signalling pathways

Increase of NMDAR function: AßOs induce neuronal oxidative stress through an NMDAR-dependent mechanism

A $\mathrm{BO}$ s bind to NMDAR $\rightarrow$ excessive activation of NMDAR $\rightarrow$ inflow of $\mathrm{Ca}^{2+}$ to neurons $\rightarrow$ excitotoxicity

$\mathrm{A} \beta \mathrm{O} \rightarrow$ synaptic dysfunction by inducing calcineurin-dependent internalization of AMPAR

Initial interaction of $\mathrm{A} \beta \mathrm{OS}$ with PrPC on the neuronal surface which leads to:

Disturbed regulation of BACE1 activity

Inhibition of elongation of $A \beta$ fibrils

Intracellular $\mathrm{Ca}^{2+}$ increase in neurons via the complex PrPCmGluR5 $\rightarrow$ impairment of synaptic plasticity

Complexes of $\mathrm{ABO}$ s with PrPC generate mGluR5-mediated influx of $\mathrm{Ca}^{2+}$ in neurons $\rightarrow$ excitotoxicity

A $B O-P r P C-m G l u R 5$ complexes signalling pathway involved in dendritic spine loss

$A \beta O$ s induce the degradation of $\beta 2 A R s$, which leads to:

Enhanced $\gamma$-secretase activity $\rightarrow A \beta$ plaque formation

Reduction of neurogenesis

Reduction of the levels of synapse-associated proteins such as synaptophysin, synapsin 1, and PSD-95

$A \beta 42$ binds to $a 7 n A C h R \rightarrow$ loss of cholinergic neurons in the brain $\rightarrow$ receptor internalization and intracellular accumulation of $A \beta$

$A \beta O s$ bind to neuronal IR $\rightarrow$ impaired insulin signalling and brain insulin resistance $\rightarrow$ elevated $A \beta$ production and reduced $A \beta O$ clearance $\rightarrow A \beta$ deposits in the brain $\rightarrow$ neuronal damage

A $\mathrm{BOs}$ bind to membrane p75NTR $\rightarrow$ formation of annular amyloid pores and ion channels $\rightarrow$ induction of aberrant cytoskeletal changes in dendritic spines

$A \beta O$ s bind to IGF-1R $\rightarrow$ phosphorylation of IGF-1R $\rightarrow$ induced p75NTR expression $\rightarrow$ cell death by fibrillary form of $A \beta$

$\mathrm{A} \beta \mathrm{O}$ s bind to $\mathrm{Pir} B \rightarrow$ impartment of synaptic plasticity $\rightarrow$ disruption of hippocampal long-term potentiation $\rightarrow A \beta$-induced deficits of memory

$\mathrm{A} \beta \mathrm{O}$ s bind to $\mathrm{F} c \gamma \mathrm{R} I \mathrm{l} b \rightarrow \mathrm{A} B \mathrm{O}$-induced inhibition of long-term potentiation $\rightarrow A \beta$-mediated neuronal dysfunction

$\mathrm{AD}$-associated TREM 2 mutations $\rightarrow$ reduction of $\mathrm{A} \beta \mathrm{O}$ s binding and degradation of $A \beta \rightarrow$ microglial depolarization, induction of $\mathrm{K}^{+}$ current into cells as well as increased cytokine expression and secretion, cells migration, proliferation, apoptosis, and morphological changes of microglia

AßOs reduce Eph receptor expression, promote its endocytosis and its degradation in the proteasome, which leads to:

Loss of dendritic spine

Synaptic dysfunction

Increase of synaptoneurosomes

Impairment of NMDAR functioning and cognitive deficits

Expression of RAGE is increased in the AD brain $\rightarrow$ RAGE is responsible of $A \beta$ influx from plasma to $B B B \rightarrow$ increase of free $A \beta$ fraction in plasma

$A \beta$ alone did not bind directly to LRP-2, whereas complexes of $A \beta-40$ with $A p o J$ are able to react with $L R P-2 \rightarrow$ clearance of $A \beta$

$1,25-(\mathrm{OH}) 2 \mathrm{D} 3$ binds to $\mathrm{VDR} \rightarrow$ increase the expression of amyloid transporters (i.e. LRP-1) $\rightarrow$ increase of transport of $A \beta$ across the $\mathrm{BBB} \rightarrow \mathrm{A} \beta$ clearance 
Table 1 (continued)

\begin{tabular}{lll}
\hline Receptor & Localization & Proposed mechanisms \\
\hline SIRT1 & $\begin{array}{l}\text { Predominantly located in the nucleus, but also in the cytosol of } \\
\text { neurons of the hippocampus and hypothalamus }\end{array}$ & $\begin{array}{c}\text { SIRT1 deficiency has been described to be responsible for the } \\
\text { increased risk of insulin resistance, obesity and diabetes, which in } \\
\text { turn are risk factors of AD }\end{array}$ \\
& $\begin{array}{c}\text { SIRT1 deficiency has been described to be involve in the reduction of } \\
\text { normal cognitive function and synaptic plasticity } \\
\text { Reduction of SIRT1 } \rightarrow \text { reduction of a-secretase activity } \rightarrow \text { enhance- } \\
\end{array}$ & ment of amyloidogenic processing of APP
\end{tabular}

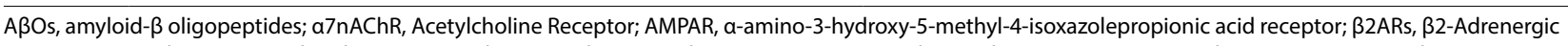
Receptors; LPR2, lipoprotein-related protein 2; mGluR5, Metabotropic Glutamate Receptor 5; EphA4, EphB2, Tyrosine Kinase Ephrin Receptors; FcyRIlb, Fragment crystallizable gamma receptor II b; IGF-1R, insulin-like growth factor 1 receptor; ILR, Immunoglobulin-Like Receptors; IR, Insulin Receptor; NMDAR, N-methyl-Daspartate receptor; p75NTR, p75 Neurotrophin Receptor; PirB, paired immunoglobulin-like receptor B: PrPC, Cellular Prion Protein; RAGE, Receptor for Advanced Glycation Endproducts; SIRT 1, Sirtuin 1; TREM2, Triggering Receptor Expressed on Myeloid Cells 2; VDR, vitamin D receptor

receptor-related protein (LRP) $[107,108]$. The peripheral administration of soluble LRP has been proposed as a promising treatment for $\mathrm{AD}$ by increasing $\mathrm{A} \beta$ efflux from the brain to peripheral blood [109]. Similarly, increasing the $A \beta$ clearance has been studied as a therapeutic option for AD. Some proteases, such as metalloproteinase 9, neprilysin, and insulin-degrading enzyme, can degrade $A \beta$ plaques. The levels of these enzymes decline in $\mathrm{AD}$ and this could contribute to $\mathrm{A} \beta$ accumulation and senile plaque formation. The activation of these types of enzymes has also been proposed as a therapeutic approach to AD [109].

Therapeutically decreasing $A \beta$ aggregation is one of the most explored routes to interfere clinically with $A D$ progression. Drugs like tramiprosate, EGCG, ELND005, and melatonin have been studied in pre-clinical and clinical trials because of their demonstrated inhibition of the aggregation of $A \beta$ peptides and dissolution of pre-formed fibrils [4]. Likewise, the use of monoclonal antibodies against $A \beta$ aggregation has been explored. For instance, aducanumab has been shown to enter the brain, bind parenchymal $A \beta$, and reduce soluble and insoluble $A \beta$ in a dose-dependent manner in both transgenic mouse models and patients with prodromal or mild AD [110]. Aducanumab is currently being investigated in a phase III clinical trial (NCT01677572) that is expected to provide compelling support for the amyloid hypothesis. However, the FDA's advisory committee recently expressed concern about the biostatistical and neurologic results of this trial. Moreover, experts agreed that the aducanumab efficacy data were weak and pointed to inconsistencies in the data that came from two futility-stopped Phase 3 trials and one Phase $1 \mathrm{~b}$ trial. Thus, the committee deemed the evidence premature for approval and recommended a confirmatory trial. The decision now rests with the FDA, which is predicted to decide by June 2021 [111].

Boada et al. investigated the effects of plasmapheresis with albumin replacement, plus intravenous immunoglobulin and obtained promising results in terms of disease progression in a multicenter, randomized, blinded and placebo-controlled, parallel-group, phase IIb/III clinical trial (AMBAR) of mild-to-moderate AD patients (NCT01561053) [112, 113]. The molecular basis of this innovative therapeutic approach was the uptake and clearance of blood $A \beta$ by replaced albumin, thereby promoting the transport of $A \beta$ peptides from CSF to plasma, which in turn reduces the $A \beta$ burden by restoring the normal balance of $A \beta$ between brain and blood. The trial obtained promising results in terms of disease progression, indicating that this approach is an important development in AD therapeutics [114].

\section{Tau-based strategies}

Targeting AD as a tauopathy is another main therapeutic approach. Soluble tau is found in neuronal cells and plays a dominant role in axonal growth and neuronal development [115]. Its importance lies in its role in regulating and stabilizing microtubules, essential structures of the cell cytoskeleton. While under physiological conditions, the phosphorylation and de-phosphorylation of tau are in equilibrium, thus maintaining its ability to bind to microtubules; in pathological situations, its hyperphosphorylation generates insoluble filaments in the form of tangles, which leads to synaptic dysfunction and neuronal degeneration [115]. Several studies have also shown a relationship between the amyloidogenic pathway and tau, demonstrating that the acceleration of tau hyperphosphorylation is promoted by soluble $A \beta$ oligomers and, in turn, $\mathrm{p}$-tau enhances the formation and aggregation of $A \beta$ plaques [116].

Therefore, targeting tau phosphorylation is another important strategy in AD therapeutics, in particular the inhibition of tau protein kinases to prevent tau phosphorylation and the concomitant microtubule instability. Of the tau protein kinases, glycogen synthase kinase 3 (GSK3) has possibly aroused the most interest [117]. Specifically, valproate, lithium, tideglusib, caffeine, and several other GSK3 inhibitors have been studied in 
pre-clinical and clinical trials as therapeutic candidates for AD tauopathy [4]. Similarly, the reduction of tau oligomerization, prevention of microtubule stabilization, and enhancement of tau degradation are other strategies currently being studied to improve AD therapy management [4].

\section{Neurotransmission}

The cholinergic system may be strongly affected in AD. Specifically, the degeneration of cholinergic neurons in the basal anterior brain has been reported to play a predominant role in the progression of the disease [118]. Much like tau hyperphosphorylation, cholinergic deficit is also related to $A \beta$. More than two decades ago, Pittel et al. showed the potentiation of the non-amyloidogenic pathway by activating the cholinergic receptors of the cerebral cortex and cerebellum and the consequent decrease in $A \beta$ formation [119].

The inhibition of acetylcholinesterase (AChE) causes an increase in acetylcholine in the synaptic space and, therefore, an increase in cholinergic activity. Indeed, three of the four marketed AD drugs are inhibitors of AChE, namely Donepezil, Rivastigmine and Galantamine. They were approved by the FDA between 1998 and 2001, and since their inception have been used in mild or moderate phases of the disease [120]. However, these drugs have poor effectiveness, with patients continuing to show progressive cognitive impairment, suggesting that these compounds have only palliative effects. In contrast, their adverse effects, due to peripheral cholinergic hyperactivity, reduce patients' adherence to the treatments and therefore limit their success $[120,121]$.

$\mathrm{AD}$ neuronal pathogenesis also extends to the glutamatergic system, especially in later stages of the disease. At the central level, glutamate intervenes in most excitatory signals and participates in several physiological processes, such as neurogenesis, synaptic plasticity, memory, and learning [122]. Glutamatergic excitotoxicity is due to a massive influx of $\mathrm{Ca}^{2+}$ ions. In $\mathrm{AD}$, this toxicity is mainly due to the overstimulation of NMDARs caused by deposits of $A \beta$ and tau tangles. Likewise, the increase in $A \beta$ levels induces a decrease in glutamate reuptake by glial cells, which translates into an increase in neuronal death [123]. Memantine, a non-competitive NMDAR antagonist, was approved for AD treatment in 2003 by the FDA and is used in moderate to severe phases. Like $\mathrm{AChE}$ inhibitors, it acts at a symptomatic level, reducing the neurotoxic effects caused by glutamatergic excitotoxicity and improving memory and learning processes. Although its effectiveness is superior to that of AChE inhibitors, it does not stop the disease progress or resolve the pathogenesis of $\mathrm{AD}[4]$.
In terms of neurotransmission, other routes related to the development of $\mathrm{AD}$ have recently come into therapeutic focus, particularly the GABAergic system, the serotonin receptor, and the modulation of histaminergic and adenosine receptors [4]. However, further research is needed to clarify the roles of these systems in AD.

\section{Oxidative stress}

Oxidative stress crucially drives many disorders, especially those inherent to the CNS, where there is very high oxygen consumption but normally low concentrations of antioxidant enzymes [124]. In 2001, Nunomura et al. reported the level of oxidative stress in the early stages of AD [125]. Unexpectedly, oxidative damage decreases with disease progression and the formation of senile plaque and tau tangles, suggesting the existence of compensatory mechanisms. Some researchers have suggested that the appearance of both senile plaque and tau tangles is enhanced with increasing oxidative stress. However, other authors have claimed that the participation of $A \beta$ in the deregulation of the intracellular homeostasis of $\mathrm{Ca}^{2+}$ and different mitochondrial mechanisms instead lead to the appearance of oxidative processes [124].

There is inconsistent evidence about the effect of exogenous antioxidants such as vitamins, carotenoids, phytochemicals, and synthetic compounds against already established oxidative stress damage. However, rutin, curcumin, and melatonin, potent antioxidant compounds, have been shown to possess several beneficial roles apart from their powerful antioxidant effects, such as amyloiddisaggregating properties and anti-inflammatory activity [126]. Likewise, boosting endogenous antioxidant activity is another strategy for the oxidative stress approach to AD. The nuclear factor 2 (Nrf2) /antioxidant response element (ARE) cascade is the primary endogenous antioxidant pathway. The translocation of Nrf2 from the cytosol into the nucleus is blocked in AD. Thus, drugs inducing the Nrf2/ARE pathway could be an interesting approach for the treatment of AD [4].

\section{Neuroinflammation}

Neuroinflammation is the physiological response of the CNS immune system against molecular alterations in the brain tissue. Growing evidence suggests that the pathogenesis of $\mathrm{AD}$ is not restricted to neurons, but also involves immunity in the brain [66]. Thus, the activation of astrocytes and microglia, resident immune cells of the CNS, is a hallmark of neuroinflammation that is observed in most neurodegenerative conditions, including AD [127]. However, the underlying molecular mechanisms remain unclear. In general, the activation of glial cells induces many biochemical and cytological changes, such as the production of ROS, the secretion of 
proinflammatory cytokines, and the degradation of neuroprotective retinoids, thus endangering the surrounding healthy neurons. Similarly, external factors, such as systemic inflammation and obesity, likely interfere with immune processes in the brain and further promote disease progression [65]. Glial cells, mainly composed of astroglia, microglia, and oligodendroglia, become activated and adopt different gene expression profiles in pathological conditions, which leads to a neuroinflammatory response [128]. This response causes an overproduction of pro-inflammatory cytokines, which gives rise to an increase of amyloidosis state, looser maintenance of the myelin that surrounds CNS axons, an increase of ROS, mitochondrial damage, and ER stress, among other effects. Neuroinflammation finally contributes to the neurotoxic processes that produce neuronal death in $\mathrm{AD}$ [128].

Epidemiological data show the protective effect of anti-inflammatory agents in neurodegenerative diseases. Thus, the hypothesis linking neuroinflammation to the pathogenesis of $\mathrm{AD}$ has gained strength in recent years, even suggesting that its onset occurs long before memory impairment becomes clinically evident [129]. This has also promoted studies of NSAIDs in AD pre-clinical and clinical trials. In the context of AD NSAIDs may not only inhibit cyclooxygenase, but also target $\alpha$-secretase or maintain $\mathrm{Ca}^{2+}$ homeostasis [4]. However, although epidemiological and observational studies have highlighted the beneficial effect of NSAIDs in reducing the symptoms and progression of $\mathrm{AD}$, randomized clinical trials and meta-analyses have failed to corroborate this significantly [130].

\section{Mitochondria damage approach}

The implications of mitochondrial dysfunction, mainly caused by ROS overproduction have also recently been described in the pathogenesis of AD [131]. Controlling ROS production is considered even more promising than traditional antioxidant strategies, which only act against accumulated ROS. Some molecules that have been studied in terms of mitochondrial anti-oxidants are coenzyme Q10, L-carnitine, triphenylphosphonium, and lipoic acid [132]. The latter also increases acetylcholine production and down-regulates the expression of pro-inflammatory cytokines [133]. Dimebon, an anti-histaminic drug, was also evaluated for its activity towards mitochondria and shown to block mitochondrial permeability transition pore opening, which translates to protection against cellular dysfunction and apoptosis processes [134]. However, the clinical translation of mitochondrial therapeutics has not yet occurred [135] and further studies are needed to elucidate the specific role of mitochondria in AD development.

\section{Metabolic alterations}

In recent years, several studies have focused on clarifying the relationship between dementia and metabolic disorders such as diabetes, obesity, dyslipidemia, and hypertension. The alteration of the insulin pathway, a reduction in the expression of its receptors, an increase in free fatty acids and cholesterol, an increase in lipogenesis, and altered vascular function are some comorbidities related to the appearance of $\mathrm{AD}$ [7]. All these processes alter the clearance of $A \beta$ in brain tissue, stimulate the hyperphosphorylation of tau, promote astrocyte and microglia activation and the secretion of ROS, and, ultimately, exacerbate the signs and symptoms of AD [136]. This highlights the importance of addressing both disorders as a single entity and the need to elucidate the underlying molecular connections to clarify the relationship between them and, therefore, their etiology. Thus, statins or antidiabetic drugs are emerging as potential, promising therapeutic candidates in AD management $[137,138]$.

\section{Controlled drug delivery systems}

Until the mid-twentieth century, the most common pharmaceutical formulations were tablets, capsules, and syrups. These types of formulations meant an average of 3-4 administrations per day for patients. Likewise, many drugs with limited solubility and bioavailability had restricted use in the clinic, regardless of the therapeutic potential of the active molecule. Controlled drug delivery systems address many of these obstacles.

In 1952, Smith Klein Beecham designed the first controlled release system (CRS) called "Spansule ${ }^{\circledR}$ technology" [139]. This resulted in the first generation (1G) of CRS, which focused on the control of release kinetics. Until the 1980s, 1G CRS were most productive, with a large number of new formulations with easy administration (mainly oral and transdermal routes) [140]. The second generation (2G) focused on developing more advanced systems, such as CRS of intelligent polymers sensitive to the environment, zero-order releases, depot formulations aimed at very long administrations (months) or hydrogels. This generation was not as productive as the $1 \mathrm{G}$ in terms of the number of formulations that entered into clinical practice, due to the complexity of development presented by these new systems [139].

It was not until 2010 that the third generation of CRS (3G) emerged. 3G CRS comprise present and future developments to improve the clinical problems noted with 2G CRS. Efforts have focused on developing CRS with more predictable kinetics through in vitro methods, selective releases, such as longer-lasting systems and easy administration, and, overcoming the physicochemical and biological barriers that were not breached by $2 \mathrm{G} \mathrm{CRS}$ [140]. Currently, the use of different nanocarriers is one 
of the most widely used state-of-the-art technologies to overcome the disadvantages of available drugs and offer new theragnostic approaches, especially in those diseases whose affected organs are difficult to access, such as AD.

The main properties that a device should possess to be a CRS are: (i) release the drug at a predetermined rate; (ii) can be administered locally or systemically; (iii) remain in the body for a specified period of time; (iv) target the carried drug to the specific site of action [141]. In addition, due to Fick's law, diffusion is the mechanism that governs the controlled release of a drug and depends on the aqueous solubility, ionization, PKa, stability, partition coefficient, and molecular weight of the drug [141].

The general classification of CRS is based on the mechanisms that govern the release process and the entrapment of the drug [142]. Thus, the main types of CRS are: (i) diffusion-controlled CRS, which act as reservoirs and monolithic systems; (ii) water penetration-controlled CRS, where osmotic and swelling processes control the release of the drug; (iii) chemically-controlled CRS, which can be designed as biodegradable reservoirs and monolithic systems or biodegradable polymer backbones with pendant drugs, where the release ratio is managed by chemical reactions between the device and the medium; (iv) responsive CRS, which act as physically or chemically responsive systems, mechanical-, magnetic-, or ultrasound-responsive systems, and biochemicallyresponsive self-regulated systems; (v) particulate CRS, which can be designed as microparticles, liposome systems, and polymer-drug conjugates, among others [142].

The most important CRS developed for nanomedicine applications are liposomes, micelles, nanoparticles, carbon nanotubes, graphene sheets, hydrogels, dendrimers, polyelectrolyte complex, and quantum dots. Table 2 summarizes the main characteristics of all CRS developed for biomedical applications [141, 142].

\section{Nanotechnologic strategies for Alzheimer's disease Nanocarriers as diagnostic tools in AD}

One of the main problems of AD is its late diagnosis due to the delayed manifestation of first clinical symptoms compared to the onset of its molecular and cellular manifestation in the brain. When the first symptoms appear, the neuronal damage is already established and irreversible. Therefore, devices that can recognize AD biomarkers in the early stages preceding memory loss and cognitive decline have attracted much interest in clinical research. Historically, the first clinical biomarker of $A D$ was the $A \beta$ peptide of senile plaques, which accumulates in the gray matter of $A D$ brains. Thus, nanodevices specific for $A \beta$ have been designed for diagnostic purposes [143].

In this context, superparamagnetic iron oxide nanoparticles (SPIONs) used with magnetic resonance imaging
(MRI) were the first attempt to use this technology for AD diagnosis. This type of nanovehicle, which could be surface modified with specific antibodies to recognize AD biomarkers, has shown elevated targeting in in vitro, in vivo, and ex vivo AD models. Thus, SPIONs coated with ganglioside carbohydrate (sialic acid), $A \beta 1-42$ antibody, and curcumin are MRI agents used to identify $A \beta$ plaques [144, 145]. Similarly, enhanced diagnosis was provided by liposomes modified with gadolinium and ET6-21, an amyloid-targeting ligand, compared to conventional MRI in an in vivo model of AD [146]. The preferred administration route for these kinds of diagnostic nanocarriers is intranasal since it is non-invasive, allows penetration across the $\mathrm{BBB}$, and avoids systemic adverse effects. A hybrid surface-modified graphene oxide with both tau and $A \beta$ antibodies linked to magnetic coreplasmonic coat nanomaterials was also developed. This nanodevice readily detected $A \beta$ and tau proteins in an in vitro model of $\mathrm{AD}$ [143].

Another diagnostic application of nanocarriers is the specific binding of disease-specific proteins. As explained above, CFS and plasma show some of the molecular alterations that occur in the brain parenchyma of $\mathrm{AD}$ patients, with levels of $A \beta$ peptides, tau, NfL, and SNAP25 , among others, increased in both. Nanocarriers could act as uptake tools to monitor these biomarkers, thus contributing to a solid diagnosis of the stage and severity of $\mathrm{AD}$ pathogenesis [143].

\section{Nanocarriers as therapeutic tools in AD}

The complexity of the therapeutic approach to AD lies not only in its unknown etiology and the lack of available effective treatments, but also in the restricted access to the affected organ. Three biological barriers control the passage of most substances into the brain and thus significantly limit drug access: the BBB, blood-CSF barrier (BCSFB), and ependymal barrier [143]. For orally administered drugs, the gastrointestinal barrier and hepatic first-pass effect must also be added [147]. Likewise, clearance mechanisms and efflux pumps significantly reduce the half-life of drugs in the body, thereby contributing to the reduction of pharmacological effectiveness [148].

Apart from accessibility problems, drugs administered to the CNS, as well as other organs, must fulfill specific biopharmaceutical characteristics that confer high bioavailability, but these are not always present. Although their pharmacological activity might be relevant, many drugs have physicochemical disadvantages, such as low solubility, short stability, and high molecular weight, that significantly reduce their bioavailability and, therefore, their final therapeutic effectiveness [149]. Additional challenges that CNS therapies face include the presence 


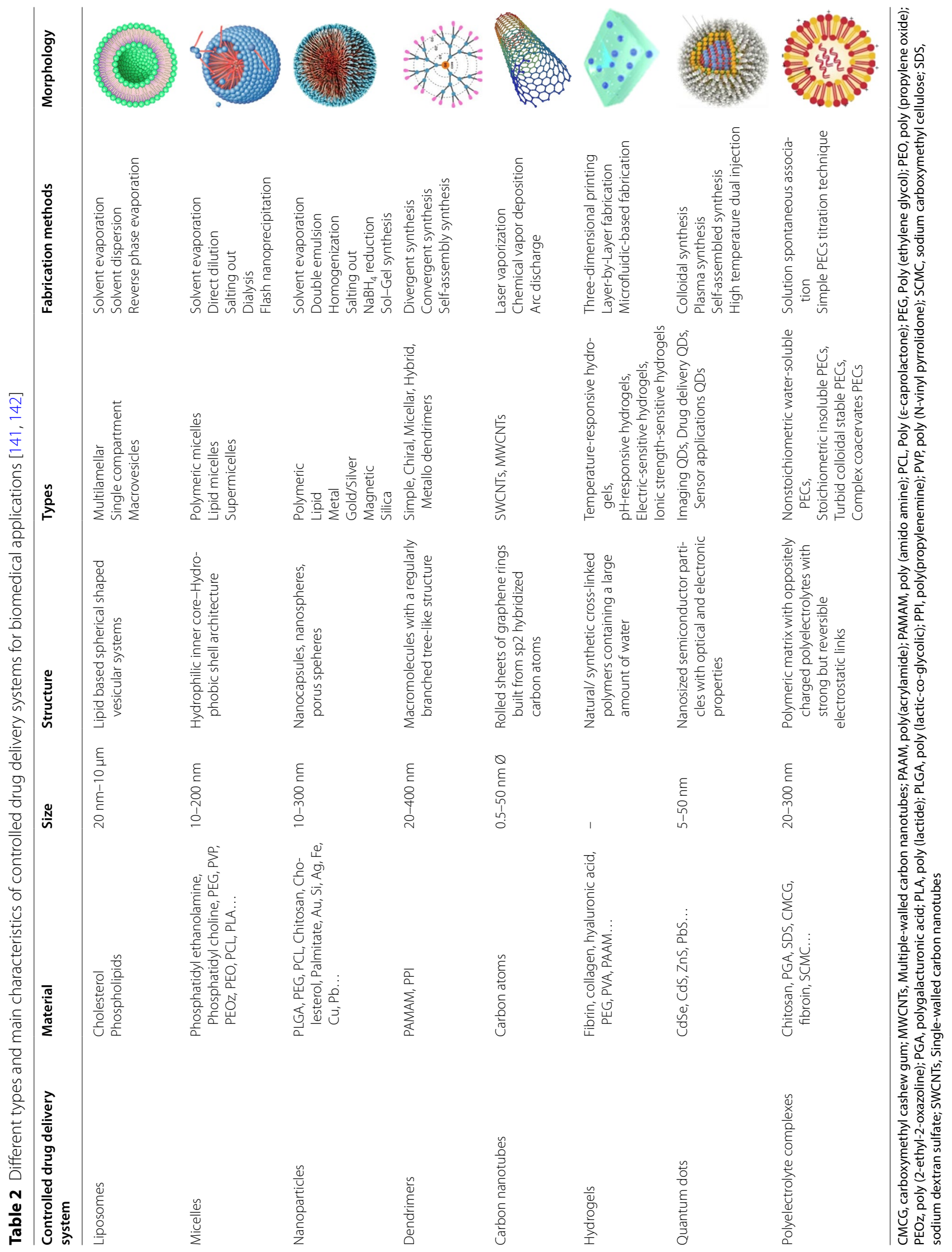


of peripheral adverse effects and the difficulty of finding the therapeutic threshold and maintaining it over time.

To overcome all these obstacles, the therapeutic potential of drug-loaded nanocarriers in different neurodegenerative diseases has been explored [150]. The high surface-to-volume ratio of nanocarriers and the possibility of surface functionalization with desired ligands are the two main exploited characteristics of these devices for drug delivery to CNS [150]. In addition, A $\beta$ targeting has been the main objective of nanomedicine to date. Three methods from nanotechnology have been used to target and modify senile plaques: (i) genetic regulation and/or inhibition of the synthesis of $A \beta$ peptide; (ii) inhibition and/or delay of the $A \beta$ nucleation dependent process; (iii) clearance of already-formed $A \beta$ plaques [143]. Álvarez-Erviti et al. employed naturally inert exosome nanocarriers to deliver BACE1-siRNA into the brain and silence BACE expression, thus inhibiting the cleavage of APP and, in turn, the overproduction of $A \beta$ peptide [151]. Recent findings related to the most common nanovehicles in preclinical assays of $\mathrm{AD}$ are described in depth below.

\section{Lipid-based nanoparticles for Alzheimer's disease}

Lipid-based NPs (LNPs) are among the most used controlled drug delivery systems to target and deliver drugs to the brain. Their safety, biocompatibility, and biodegradability make these systems an interesting choice for drug nanocarriers. Furthermore, since their inherent lipidic structure is similar to the lipid composition of the $\mathrm{BBB}$, their penetration into the brain by the transcellular pathway is favored [152]. The core of LPNs is commonly composed of monoglycerides, triglycerides, fatty acids, fatty alcohols, mixtures and waxes [153]. The most common LNPs are liposomes, solid lipid NPs (SLN), and nanostructured lipid carriers (NLCs), which mainly differ in the disposition of the lipid layers, morphology, loading capacity, average particle size, and electrokinetic behavior [154]. This type of nanocarrier can encapsulate both hydrophilic and hydrophobic drugs, which significantly increases their versatility [155]. However, their low stability, complex fabrication method, unexpected polymorphic transitions, and drug release during storage are some disadvantages that complicate large-scale production and, therefore, widespread use [154]. However, many efforts are being made to improve these issues and the potential of LNPs as diagnostic and therapeutic tools in AD and other CNS diseases [156]. Recent preclinical studies have produced interesting results by combining state-of-the-art LNPs and AD animal models (Table 3).

Almuhayawi et al. designed pomegranate extractloaded LNPs to evaluate their effects in an aluminum chloride-induced rat model of AD [157]. Pomegranate extract is highly enriched in alkaloids and tannins, which have powerful antioxidant effects. The results showed that, compared to untreated controls, animals treated with such LNPs exhibited decreased NFTs and A $\beta$ deposits, improved cognitive test scores, and increased biomarkers of antioxidant activity in the brain homogenates. Similarly, Giacomeli et al. investigated the neuroprotective effects of curcumin-loaded LNPs in a mice model of AD [158]. The therapeutic potential of curcumin has been widely recognized for many diseases, but its low solubility and, consequently, reduced bioavailability limit its pharmacological effect [159]. In this study, the authors developed a hybrid nanocarrier of a lipidic core with a PLC coat to confer better solubility. Animals treated with this nanocarrier showed improved spatial memory and reduced neuroinflammation biomarker levels in serum and the hippocampus and cortex homogenates. Pinheiro et al combined natural products and LNPs. The bioactive load was quercetin, a flavonoid present in many vegetables and fruits with strong antioxidant activity. The authors went one step further and coated the LNPs with transferrin to enhance transport across the BBB [160]. These LNPs were found to be non-toxic for hCMEC/D3 cells, a frequently used in vitro model of human $\mathrm{BBB}$, and to enhance the penetration of quercetin through the BBB. Moreover, these LNPs inhibited A $\beta 1$ 42 fibril formation in an in vitro model [161]. Similarly, $\alpha$-bisabolol, a sesquiterpene alcohol found in Matricaria chamomilla essential oil with demonstrated anti-plasmodial, anti-microbial, anti-inflammatory, anti-cancer and anti-cholinesterase properties, has significantly reduced bioavailability due to low solubility [162]. Sathya et al. developed $\alpha$-bisabolol-loaded Cholesterol LNPs and explored their neuroprotective effects in an $A \beta$-induced in vitro model of Neuro-2a cells, a fast growing mouse neuroblastoma cell line [163, 164]. These studies show that such LNPs possess antioxidant potential, significantly reduce $\beta$-secretase, caspase- 3 , and cholinesterase activities, inhibit $A \beta$ aggregation, and protect Neuro2a cells from $A \beta$-induced neurotoxicity by reducing the expression of Bax and inducing the expression of Bcl-2 proteins $[163,164]$.

Finally, Dara et al. recently investigated the therapeutic potential of erythropoietin (EPO) in AD. EPO is neuroprotective in several diseases, such as spinal cord injury, cerebral ischemia, epilepsy, and diabetic neuropathy [165]. Moreover, EPO contributes to neuronal survival and the regulation of neurogenesis in both Parkinson's disease and AD [166, 167]. However, EPO has restricted penetration through the $\mathrm{BBB}$ due to its hydrophilicity, rapid clearance from the bloodstream, and high molecular weight. Encapsulated in SLNs, EPO reduced oxidative stress and $A \beta$ deposition in the hippocampus more 


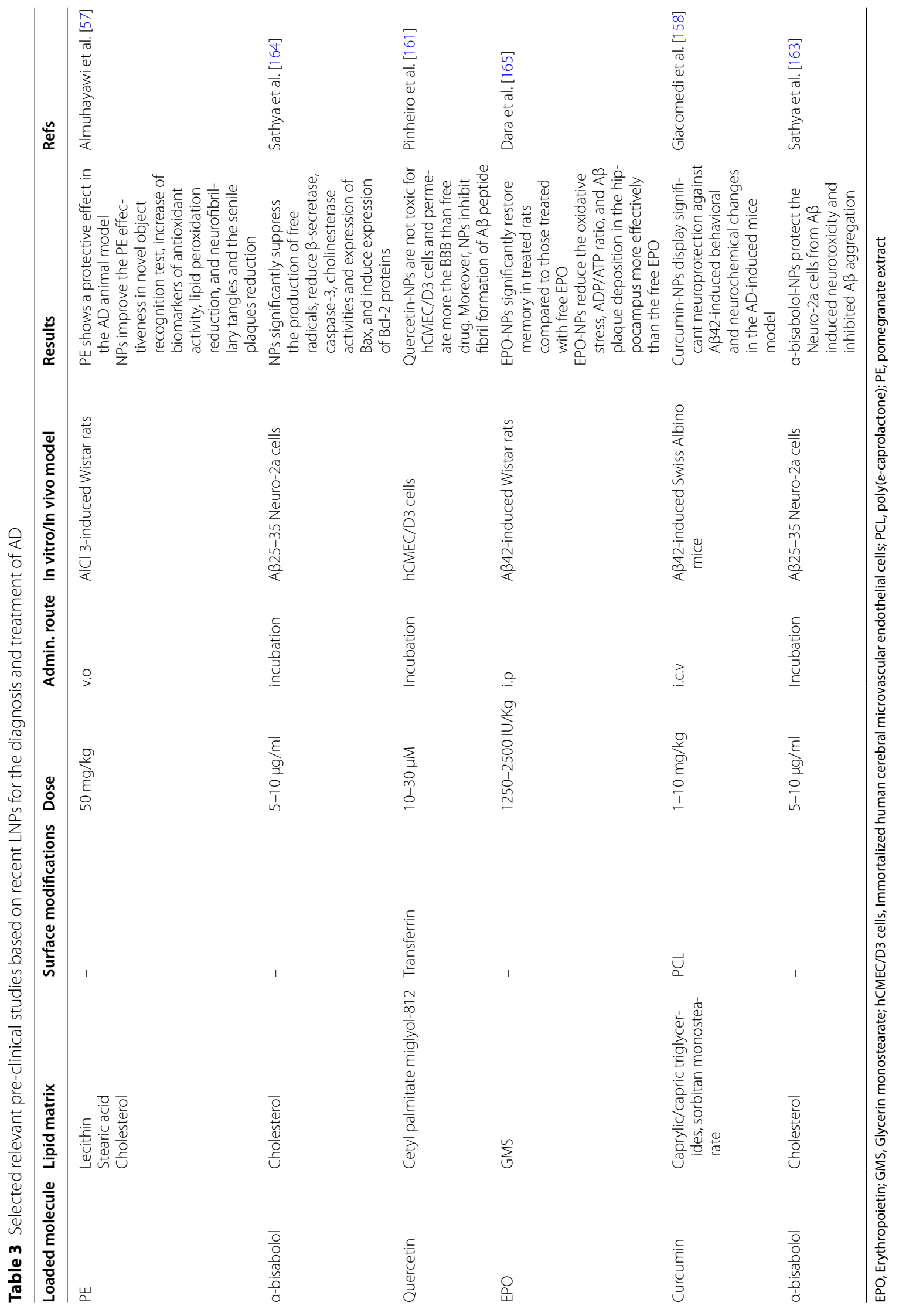


efficiently in an A 342 -induced Wistar rat model of $A D$ [165]. Moreover, animals treated with EPO-LNPs exhibited increased spatial memory compared to free EPOtreated littermates.

\section{Polymeric-based nanoparticles for Alzheimer's disease}

Due to their versatility and ease of fabrication by different methods, polymeric NPs (PNPs) are among the most used carriers in nanomedicine applications $[168,169]$. Their mean average size is between 10 and $1000 \mathrm{~nm}$ and they can load high amounts of both hydrosoluble and hydrophobic drugs [170]. Depending on the polymer composition, which can be natural or synthetic, PNPs can have both positive and negative surface charges. This characteristic significantly conditions their biological behavior, muco-adhesiveness, and penetration [171].

PNPs can be formulated as nanocapsules and nanospheres. Nanocapsules possess a vesicular structure in which drugs are dissolved in a liquid core surrounded by the polymeric capsule. In contrast, nanospheres are composed of a polymeric matrix in which drugs are dispersed in the matrix gaps or adsorbed onto the sphere surface [172]. The polymers most used to manufacture PNPs are polylactide (PLA), poly(lactide-co-glycolide) (PLGA), chitosan, polyethyleneimine (PEI), and poly- $\varepsilon$ caprolactone $(\mathrm{PCL})$, all of which were approved by the FDA for biomedical applications twenty years ago [173]. These kinds of vehicles have many important advantages, such as their high loading capacity, controlled drug release kinetics, surface modifications for brain targeting, safety, biodegradability, biocompatibility, and easy elimination [168]. However, these systems also have some disadvantages that limit their use, especially the need for organic solvents during their fabrication. Nevertheless, their pharmacological potential has encouraged many researchers to explore the potential of PNPs for the diagnosis and therapeutic management of different neurodegenerative diseases [150]. Table 4 summarizes recent advances on the preclinical evaluation of state-of-the-art PNPs in AD.

The lack of effective treatments for $\mathrm{AD}$ has led to an incessant search for new pharmacological alternatives. Among these alternatives, natural compounds are emerging as potential therapeutic drugs for several diseases, such as cancer, diabetes, and cardiovascular and neurodegenerative diseases, including $\mathrm{AD}$. Thus, Dhas et al. developed cationic biopolymer core/shell NPs loaded with lutein (LT), a natural dietary carotenoid mainly obtained from food such as green vegetables, eggs, and corn [174]. Although this molecule has shown promising beneficial effects, such as anti-inflammatory, antioxidant, and anti-cancer activity, its reduced solubility and bioavailability have restricted its use in the food and pharmaceutical industries [175]. In this study, the authors demonstrated that the developed nanocarrier possesses the optimal physicochemical characteristics for suitable in vivo administration. The PNPs have good entrapment efficiency, sustained LT release, protection of LT integrity, biocompatibility with brain cellular models, and efficient passage through an in vitro BBB [174]. Moreover, after intranasal administration in rats, LT-loaded PNPs accumulated in the brain and exhibited reduced toxicity and significant ROS scavenging activity [174]. Similarly, Sathya et al. evaluated the therapeutic potential of phytolloaded PNPs in the regulation of the expression of ADrelated genes and neuronal degeneration in both in vitro and in vivo models of AD [176]. As with LT, phytol is a natural compound with several pharmacological properties but poor solubility and low absorption, resulting in reduced bioavailability that limits its clinical use [177]. In the in vitro Neuro-2a cell model, phytol-loaded PNPs inhibit apoptosis-mediated cell death and cholinesterase activity. In a transgenic nematode AD model, these PNPs were found to increase chemotaxis and lifespan and reduce ROS production and $\mathrm{A} \beta$ deposition. Furthermore, PNP treatment upregulated a gene involved in the longevity of nematodes, downregulated the expression of several $\mathrm{AD}$-associated genes, and reduced the expression of $A \beta$ peptide at the protein level [176].

Cano et al. also studied natural-compound-loaded PNPs for AD [178]. In this study, the selected molecule was EGCG (see also above), which has shown therapeutic activity in many diseases, such aas breast cancer, diabetes mellitus, Down syndrome, and different neurodegenerative diseases, but its instability in water solutions and in vivo administrations reduce its effectiveness [179]. Co-encapsulating EGCG into PEGylated PLGA NPs with ascorbic acid (AA) to prevent auto-oxidation produces a nanosystem with significantly enhanced EGCG integrity, which is correlated with improved EGCG effectiveness in a variety of biological assays relevant to $\mathrm{AD}$ [178]. EGCG/ AA PNPs readily cross the BBB both in vitro and in vivo. Moreover, compared to free EGCG, EGCG/AA NPs exhibit improved bioavailability and pharmacokinetic profile and, in an $\mathrm{AD}$ mouse model, improved memory learning processes, reduced cognitive decline, neuroinflammation, and $A \beta$ plaque burden, and increased synaptic expression.

Taken together, these findings highlight the relevance of natural compounds as promising therapeutic strategies in AD management. However, PNPs loaded with natural compounds have not only been studied as therapeutics, but also for diagnostic purposes. In this regard, Ahlschwede et al. developed curcumin-loaded PLGA NPs functionalized with K16ApoE, a BBB penetration 


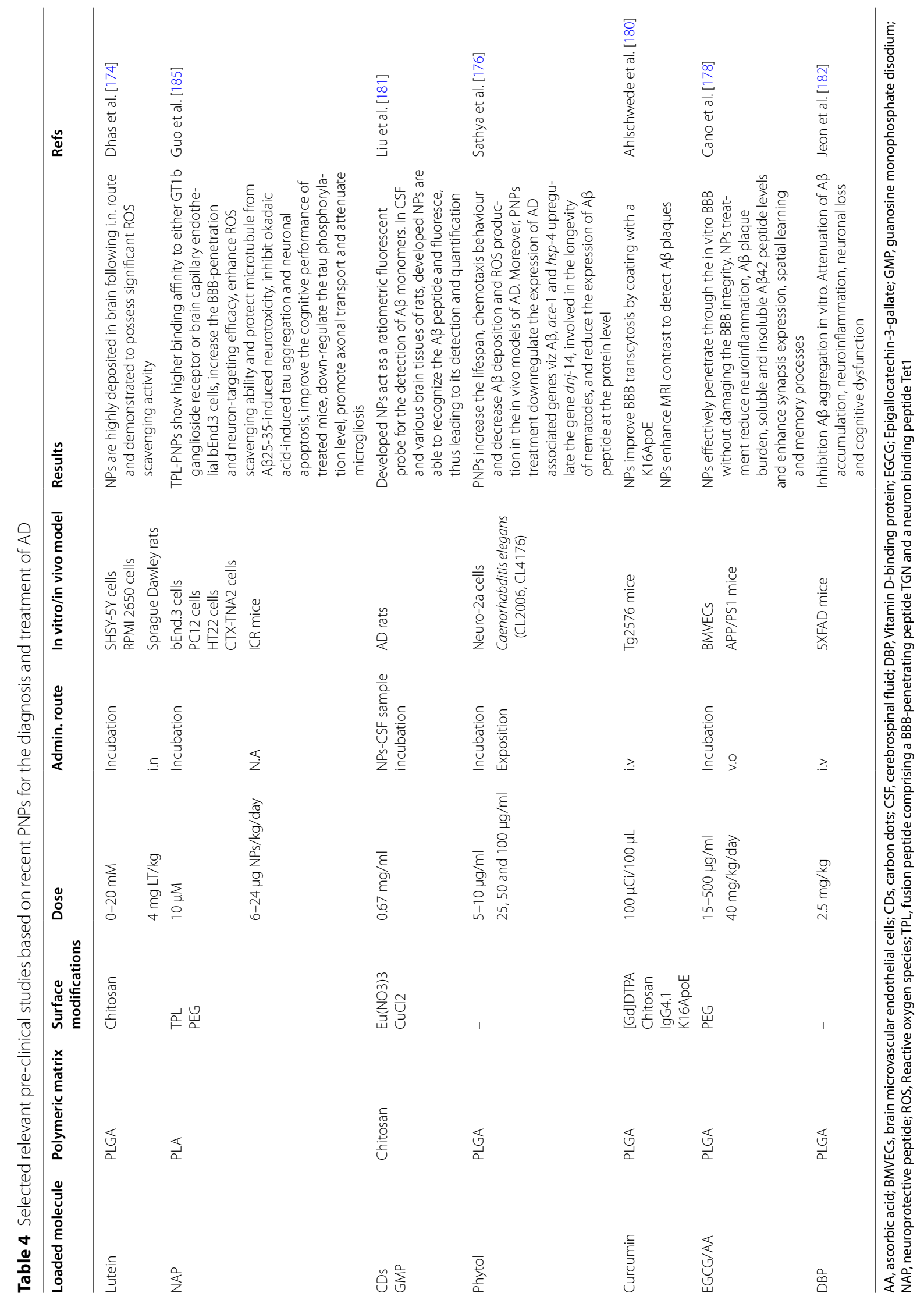


peptide. The authors aimed to create an effective tool for detecting cerebrovascular $A \beta$ and treating cerebral amyloid angiopathy, which is widely observed in AD development [180]. Using a quartz crystal microbalance with dissipation monitoring technology, the authors found that developed PNPs effectively migrated from the blood flow to the vascular endothelium. As expected, K16ApoE coating significantly improved BBB transcytosis and provided specific MRI contrast to detect brain $A \beta$ plaques. Moreover, K16ApoE-PNPs also showed specific targeting of vasculotropic DutchA $\beta 40$ peptide that accumulated in the cerebral vasculature. Despite this study's innovations, in vitro and in vivo assays are still required to describe the therapeutic effects of curcumin-loaded K16ApoEPNPs on memory impairment, cognitive decline, and molecular alterations related to AD development. Liu et al. used nanotechnology for diagnosis. The authors designed carbon dots (CDs) sensitized lanthanide infinite coordination polymer (ICP) NPs and a PNPs-based ratiometric fluorescent probe mainly composed of CDs, Europium Nitrate $\left(\mathrm{Eu}\left(\mathrm{NO}_{3}\right)_{3}\right)$, and $\mathrm{Cu}^{2+}[181]$. The detection technique was based on the competitive coordination interaction of $\mathrm{Cu}^{2+}$ between the guest $C D$ s and $A \beta$ monomer. Briefly, in the absence of $A \beta$, the coordination interaction between $\mathrm{CDs}$ and $\mathrm{Cu}^{2+}$ disrupts the antenna effect, leading to the fluorescence quenching of $\mathrm{Eu}^{3+}$. When developed PNPs contact an $A \beta$-enriched sample, the stronger coordination between $\mathrm{A} \beta$ monomer and $\mathrm{Cu}^{2+}$ restores the red fluorescence of $\mathrm{Eu}^{3+}$, leading to the detection and quantification of $\mathrm{A} \beta$ peptide by fluorescence. In this study, developed PNPs were exposed to CSF/brain tissue samples of AD rats. The authors showed that their method is highly sensitive for the in vivo analysis of $A \beta$ monomer, thereby demonstrating the utility of this ratiometric fluorescent probe.

Some endogenous substances have been also described as pharmacological tools for AD. In that sense, Jeon et al. explored the therapeutic potential of Vitamin D-binding protein (DBP) [182]. DBP is a glycoprotein that is highly expressed in a wide variety of cells and tissues and has been shown to play important roles in several physiological processes. Likewise, several studies have highlighted that DBP levels are altered in the serum and CSF of AD patients and possess optimal properties for $A \beta$ binding, and thus peripheral clearance $[183,184]$. However, previous studies have shown that plasma DBP has a relatively short half-life. Jeon et al. aimed to encapsulate DBP into PLGA matrices to prolong the presence of DBP in the bloodstream and evaluate their effectiveness in an $\mathrm{A} \beta$-overexpressing mice model of $\mathrm{AD}$. The developed nanocarrier inhibited the polymerization and accumulation of $A \beta$ in both in vitro and in vivo models, ameliorated $A \beta$-associated neuroinflammation and neuronal loss, and significantly reduced the cognitive impairment of treated transgenic mice.

Finally, Guo et al. recently developed a PNP coated with both a BBB-penetrating ligand and a neuron-targeting ligand for carrying the neuroprotective peptide NAP. NAP has been shown to provide neuroprotective activity against NMDA receptors and $A \beta$-mediated excitotoxicity by interacting with glial and neuronal tubulin, thereby promoting microtubule assembly and the protection of the neuronal cytoskeleton [185]. As explained above, the clinical application of NAP is restricted due to its enzymatic degradation, ineffective neuron targeting and reduced half-life in the bloodstream [185]. The encapsulation of NAP in the nanocarrier improved its stability in vivo. Likewise, coating the PNP's surface effectively increased the accumulation of developed NPs in brain neurons. The evaluation of its pharmacological properties highlighted that NAP-PNPs promote the alleviation of oxidative stress, microtubule disruption, the attenuation of neuroinflammation, and the inhibition of tau aggregation and apoptosis processes. Likewise, a rescue of the memory deficits and spatial learning in AD mice was observed after PNP treatment. Moreover, treatment with these PNPs also prevented tau hyper-phosphorylation and restored axonal transport.

\section{Metal-based nanoparticles for Alzheimer's disease}

Metal-based NPs (MNPs) are the most relevant inorganic nanocarriers because of their widespread use in nanomedicine applications [186]. These vehicles usually have an average size of 10 to $100 \mathrm{~nm}$. Moreover, their high surface area allows for coating with different molecules (e.g., antibodies, genes, peptides), which lets them act as biosensors and targeting tools. MNPs are commonly fabricated with gold, silver, iron, zinc, and copper, all of which provide different properties to the final nanocarrier [187]. MNPs have demonstrated anti-microbial, anticancer, anti-inflammatory, and anti-oxidant properties, among others [188].

Different methods are employed in the fabrication of MNPs, with top-down methods (e.g., mechanical milling, laser ablation, and ion sputtering) and bottom-up methods (e.g., solid-state methods, liquid-state synthesis methods, gas-phase methods, and electrochemical deposition). Note that the selected fabrication method significantly conditions the final physicochemical properties, morphology, and long-term stability of MNPs [189]. Green synthesis methods, an emerging trend of nanotechnology, can overcome the toxicity problems, high cost, and reaction complications of conventional fabrication methods, so they have attracted much recent interest. Green chemistry incorporates novel techniques to reduce the health and environmental burdens of 
conventional techniques. Some of the most used green synthesis methods involve biological methods with different microorganisms and their enzymes and using plant extracts. The main advantages of these novel techniques are reduced costs, ease of scaling up for large-scale production, and the complete elimination of energy, high pressure and temperature, and toxic chemicals [190].

Much current research focuses on MNPs because of their multiple benefits and versatility for use in disease diagnosis and treatment, labeling optoelectronic recorded media, cosmetics, and sensor technology, among others [189]. In addition, the magnetic properties of some of these MNPs can be exploited to enhance the accumulation of these NPs in specific organs since they can be detected and manipulated by remote magnetic fields [191]. Table 5 displays relevant studies involving the latest smart MNPs and AD preclinical models.

Currently, using MNPs for diagnosis purposes is being widely studied. For example, da Silva et al. recently developed a highly sensitive AChE electrochemical biosensor based on an electrode modified with iron oxide NPs and a deep eutectic solvent [192]. Enzyme-modified electrode sensors offer a powerful tool for real-time diagnostics that overcomes the drawbacks of traditional analytical approaches, such as time-consuming processes, high cost for measurements, and the need for highly specialized staff [192]. Likewise, their capability to promote the fast electron-transfer kinetics of iron oxide NPs means they had optimal properties for use in the developed biosensor. Da Silva et al. immobilized AChE on a polymeric-based film pre-formed in modified electrodes, which was composed of iron oxide NPs and an acid eutectic dissolvent. They tested different acids to evaluate their influence on the rate of growth and electrochemical properties of the polymer films and finally selected $\mathrm{HNO}_{3}$. The authors used chronoamperometry to investigate the catalytic activity of the developed biosensor for ACh detection. After exposing the biosensor to synthetic urine containing known concentrations of $\mathrm{ACh}$, the authors recorded the typical current-time voltage curves and demonstrated that this MNPs-based biosensor has high sensitivity, excellent reproducibility, and long-term stability, with significant recovery ratios. In a related study, Xing et al. investigated the development of an electrochemical technique for detecting $A \beta$ based on silver NPs aggregates as the redox reporters and a cellular prion protein $\left(\operatorname{PrP}_{95-110}\right)$ as the bio-receptor [193]. The fundamentals of their technique are based on the oscillation of the electrochemical signal promoted by the interaction of $\mathrm{A} \beta$ with the $\mathrm{PrP}_{95-110}$-MNPs. The specific binding of the $A \beta$ oligomers to the PrP peptide blocks the aggregation of the MNPs' main electro-transmitter, which decreases the electrochemical signal that can be detectable and quantifiable. The exposure of this nanodevice to both $A \beta$-enriched artificial CSF and the human serum of AD patients demonstrated that the developed detection method exhibits high sensitivity and specificity and requires simple manipulation, less time expense, lower expenses, and lower detection limits than those achieved by previously reported methods.

The use of MNPs as therapeutic tools for AD is also important. For example, Liu et al. explored the therapeutic potential of quercetin-modified gold-palladium NPs to promote the clearance of intracellular $A \beta$ by autophagy induction and, consequently, reduce $\mathrm{A} \beta$-induced neurotoxicity [194]. As explained above, quercetin is a potent natural antioxidant with limited ability to cross the BBB and easy elimination. Recent studies have shown that quercetin can accelerate the elimination of abnormal $A \beta$ by enhancing the autophagy effect of brain cells [194]. Liu et al. reported that the developed MNPs are not toxic in both in vitro and in vivo and effectively cross the in vitro BBB. Furthermore, they increased intracellular autophagy levels, promoted the degradation of autophagosomes, enhanced $A \beta$ clearance in brain cell cultures, and reduced $A \beta$-induced cytotoxicity. Similarly, Gao et al. recently evaluated multi-targeted chondroitin sulfate /selenium MNPs and evaluated their protective effect against $A \beta$-induced neurotoxicity [195]. Chondroitin sulfate is a glycosaminoglycan with several biological functions, such as antioxidation, anti-inflammation, and neuroprotection. Moreover, chondroitin sulfate is involved in cell migration, neurogenesis, axon growth, synaptic plasticity, neuron regeneration, the inhibition of $A \beta$ fibril formation, and the blocking of $A \beta$-induced cell apoptosis [196]. Also, selenium is a potent antioxidant that plays important roles in detoxification, the protection of the immune system, and the regulation of cellular redox homeostasis. Furthermore, it also inhibits the formation of $A \beta$ plaques and degrades preformed $A \beta$ fibers [197]. In this new study [195], Gao et al. obtained promising results. Their MNPs could reduce damage to the cytoskeleton and neuronal cells and oxidative stress, inhibit the aggregation of $A \beta$ and protect cells from its neurotoxicity, and attenuate the hyperphosphorylation of tau protein. Sonawane et al. also explored the effect of MNPs in Tau-related pathogenesis. The authors biologically synthesized two different protein-capped MNPs, (i) iron oxide NPs and (ii) cadmium sulfide NPs, and evaluated their effectiveness in an in vitro model of AD [198]. The surface modification of these MNPs with proteins was performed to inhibit Tau protein aggregation. To complete the biological synthesis of these carriers, two fungal species were used, Fusarium oxysporum and Verticillium sp. The composition and synthesis of both NPs were different: iron oxide NPs were synthesized 

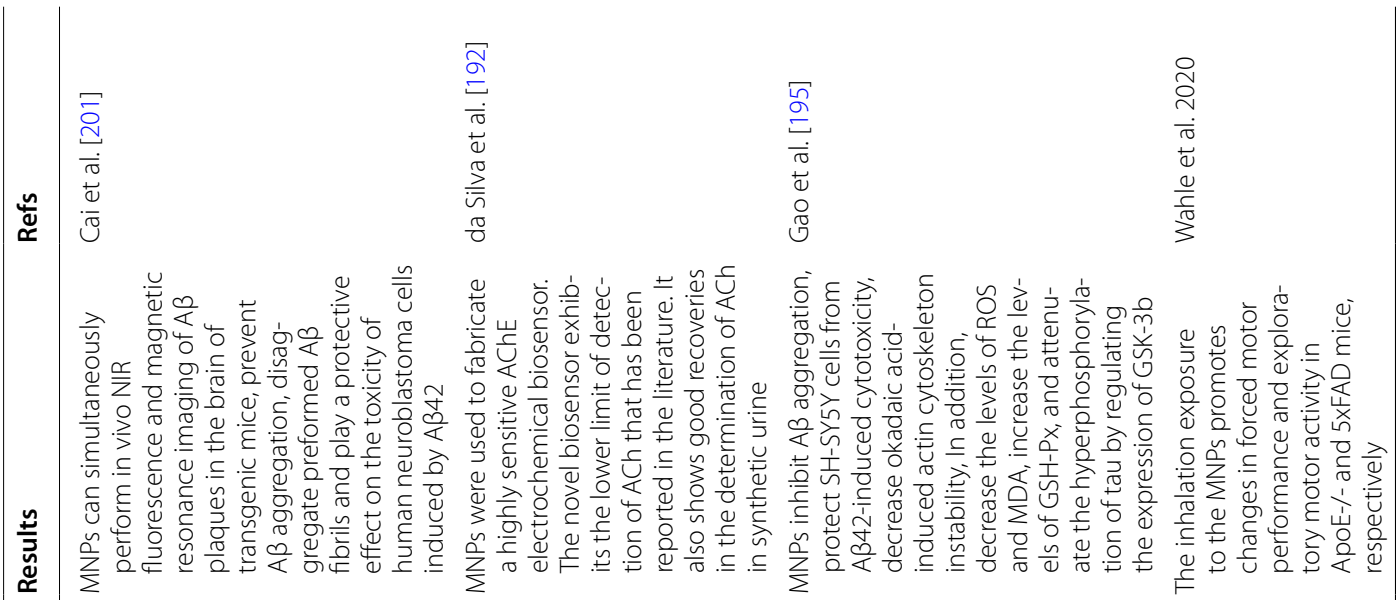

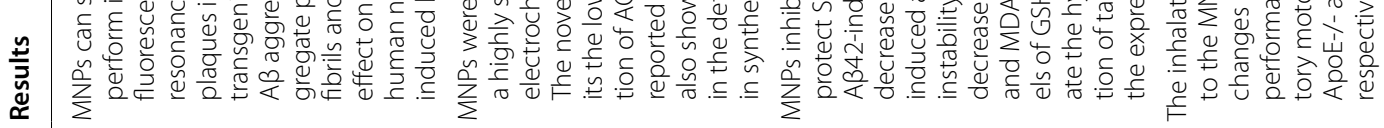

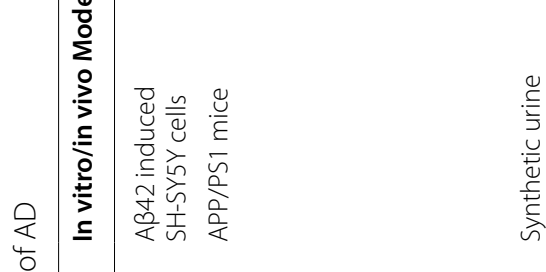

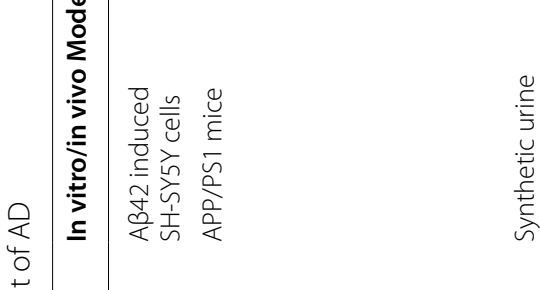

๘

离

竞

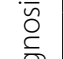

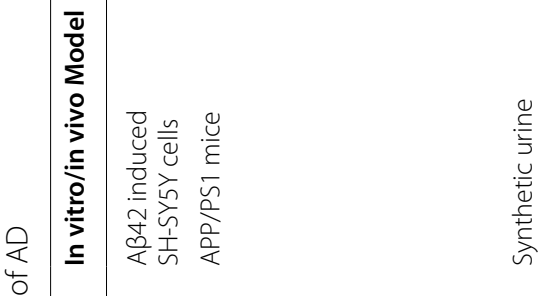

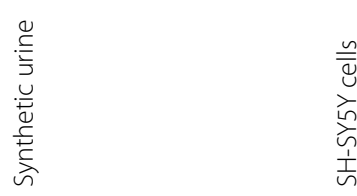

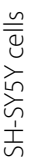

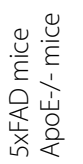

$\stackrel{s}{z}$

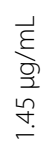

$\subseteq$

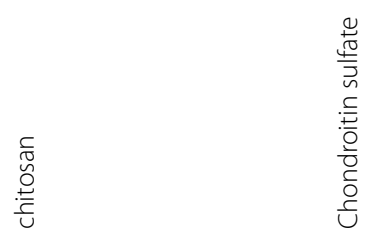

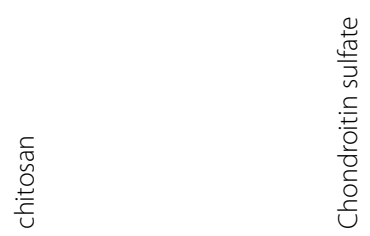

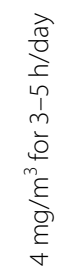

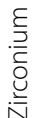

$\frac{0}{0}$
$\frac{0}{\partial}$
$\stackrel{\check{0}}{\underline{0}}$

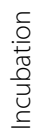

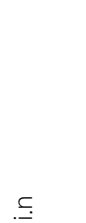

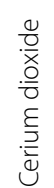




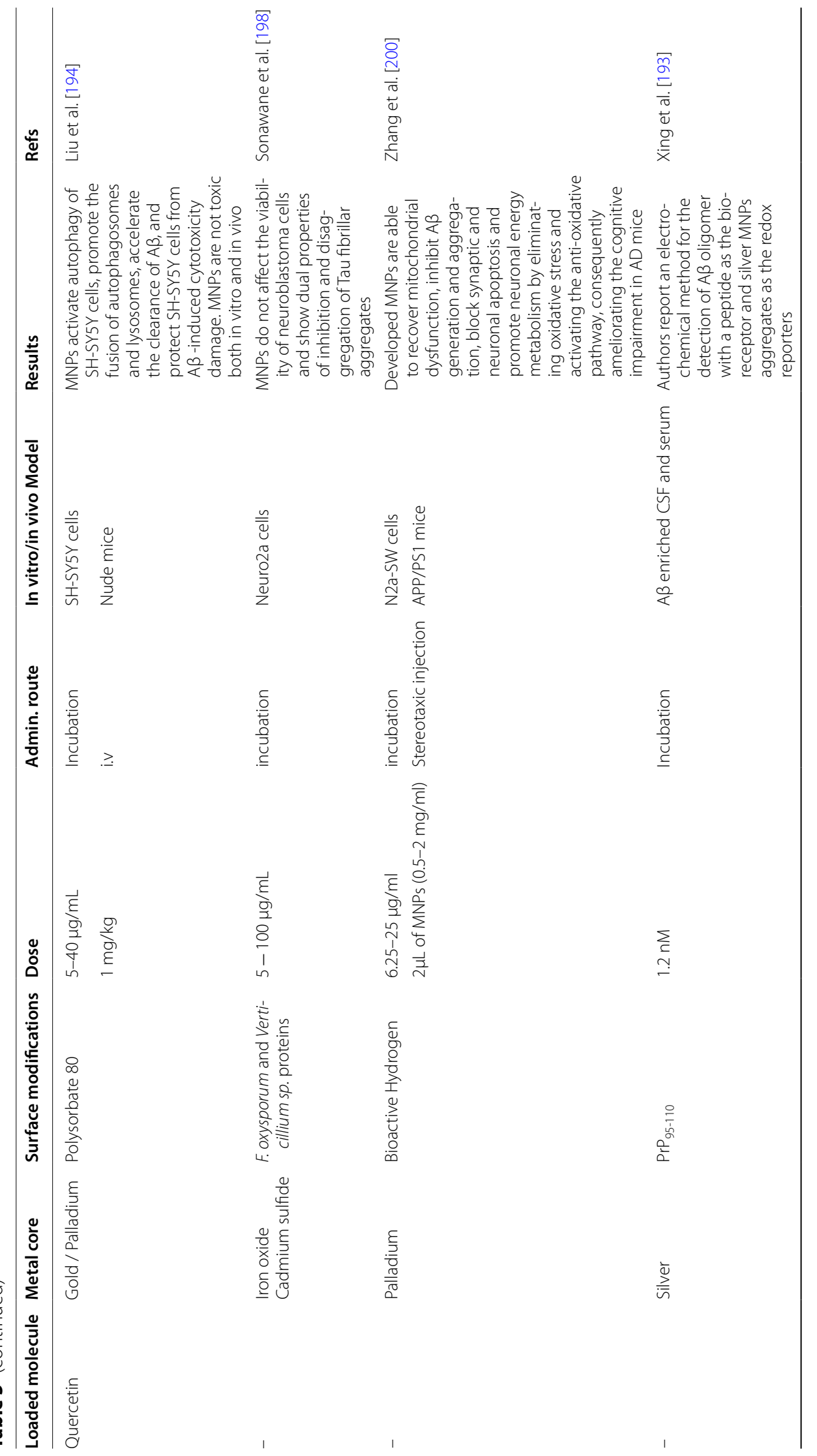




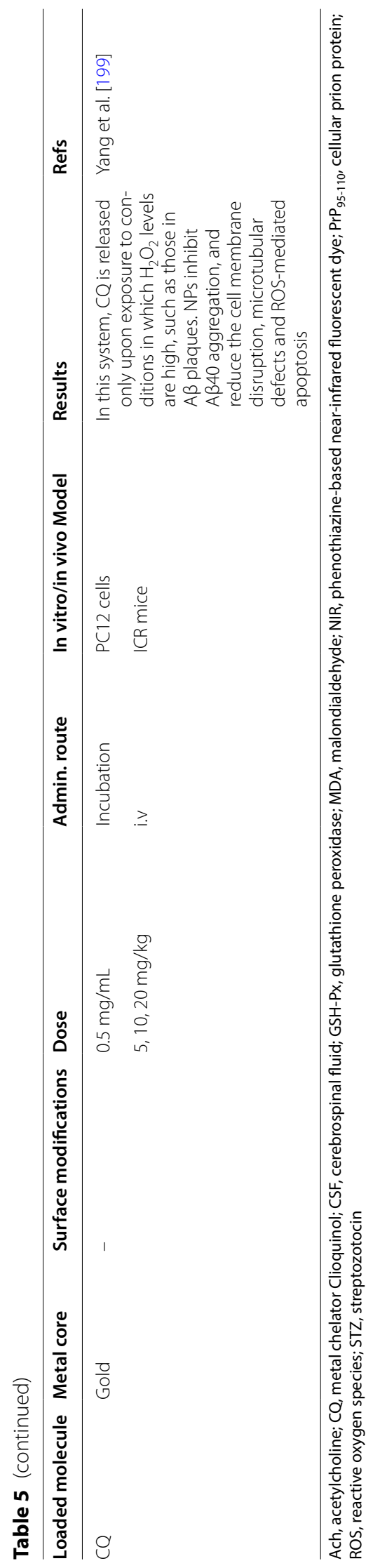


extracellularly by the fungal species with transient ferromagnetic properties and capped with hydrolytic proteins. In contrast, cadmium sulfide NPs were synthesized from the extracellular sulfate-reducing enzymes secreted by the fungus when provided with mixture of salts, so the surface proteins were composed of a mixture of four different proteins that probably belonged to the group of these sulfate-reducing enzymes. Both nanocarriers did not affect the viability of cultured cells. Furthermore, both MNPs efficiently inhibited Tau aggregation, and cadmium sulfide NPs exhibited a significant dissembling of Tau NFTs.

As described above, oxidative stress is one of the most important molecular hallmarks in AD development. In AD pathogenesis, the exacerbated formation of $\mathrm{H}_{2} \mathrm{O}_{2}$ is favored by generalized brain oxidative stress. Metal chelators can block the detrimental effects of ROS, but their non-specific interactions with metal ions of normal cellular processes and their BBB-reduced permeability significantly limit their therapeutic success [199]. Yang et al. designed an $\mathrm{H}_{2} \mathrm{O}_{2}$-sensitive detection system composed of Clioquinol-doped gold NPs-capped mesoporous silica [199], and revealed that the conjugation of ClioquinolMNPs on the surface of mesoporous silica leads to selective and sustained Clioquinol release under an increased $\mathrm{H}_{2} \mathrm{O}_{2}$ environment (e.g., surrounding of $A \beta$ plaques). Furthermore, their nanodevice efficiently crossed the $B B B$, decreased $A \beta$ self-assembly, and reduced microtubular defects, cell membrane disruption, and ROS-mediated apoptosis induced by $\mathrm{A} \beta 40$.

Hydrogen $\left(\mathrm{H}_{2}\right)$ has been shown to selectively scavenge highly cytotoxic ROS. Furthermore, Hydrogen's bio-safety and bio-diffusibility position it ahead of many compounds in $\mathrm{AD}$ drug discovery. However, the high solubility of $\mathrm{H}_{2}$ and traditional hydrogen administration routes, such as the oral intake of hydrogen-rich water, inhalation of hydrogen gas, or injection of hydrogen-rich saline, do not ensure its accumulation in the brain for the time necessary to exert its activity [200]. Zhang et al. developed reactive hydrogen-doped palladium NPs and evaluated their effects against oxidative stress-induced mitochondrial dysfunction in both in vitro and in vivo models of AD [200]. This was the first time that a nanosystem was shown to be able to perform a self-catalysis of carried $\mathrm{H}_{2}$ and realize an in situ sustained release of bio-reductive hydrogen. This innovative system effectively stores and releases bio-reductive hydrogen to selectively scavenge $\mathrm{OH}$ in $\mathrm{AD}$ cells, inhibit $\mathrm{A} \beta$ generation and aggregation, reverse synaptic deficits and neuronal death, and ameliorate mitochondrial dysfunction and cognitive impairment in transgenic AD mice.

Going a step further, Cai et al. recently developed ultrasmall superparamagnetic iron oxide NPs (USPIONs) coupled to a phenothiazine-based near-infrared (NIR) fluorescent dye for both the diagnosis and treatment of AD [201]. This compound was shown to effectively inhibit the self-aggregation of $A \beta$ and disaggregate preformed $A \beta$ fibrils, as well as exhibit high fluorescence enhancement upon binding to aggregate $A \beta$ proteins [201]. Thanks to their small size, amyloidogenic proteins, such as $A \beta$ peptides, tend to be absorbed on the surface of USPIONs, thereby reducing circulating $A \beta$. Likewise, brain cells have been shown to be highly sensitive to USPIONs and their responsiveness to an external magnetic field is an important advantage to promote brain cell uptake. Moreover, the moderate heat emitted from USPIONs under the action of a low radiofrequency field represents a significant advantage since it increases the BBB's permeability without disturbing its integrity [201]. Cai et al. revealed that developed USPIONs can prevent $A \beta$ aggregation, disaggregate preformed $A \beta$ fibrils, simultaneously perform in vivo NIR fluorescence and MRI of $\mathrm{A} \beta$ plaques in the brain, and perform a neuroprotective effect against $\mathrm{A} \beta 42$-induced toxicity.

Importantly, not only is the theragnostic potential of MNPs being studied, but also their potential neurotoxic effects. Increasing evidence shows the relevance of toxins in AD development, such as ultrafine air pollution particles. Therefore, and since nanomedicine has emerged as a promising alternative for $\mathrm{AD}$ management, concerns have been raised about the potential neurotoxic effects of different nanocarriers, especially MNPs. Their small size and easy penetration into human tissues by different access routes allow their high accumulation in the brain. Cerium dioxide NPs have gained much interest in recent years because of their radical-scavenging properties. For these reasons, Wahle et al. recently evaluated the neurotoxic effects of zirconium-doped cerium dioxide NPs in two different mice models of AD [202]. The continuous inhalation of these nanocarriers promotes changes in exploratory motor activity and forced motor performance in AD mice and increases GFAP expression in healthy mice. Thus, although MNPs represent a promising therapeutic alternative, these results highlight the importance of exhaustive research into their neurotoxicity to ensure therapeutic safety.

\section{Conclusions}

Dementias, including $\mathrm{AD}$, are the fifth-leading cause of death worldwide, accounting for 2.4 million deaths yearly. The expected increase in the number of cases of dementia in the next few decades is even more important, given that there are currently no effective diseasemodifying treatments for AD [1]. In addition, even when clinical trials are initiated, many of them fail to discover a new disease-modifying drug to market: 
of every 100 molecules in clinical AD trials, only one reaches the market, compared to the pharmaceutical industry average ratio of 14.6:1 [1]. Without novel promising treatments in sight, increasing numbers of cases will pose an undue burden on patients who have dementia, their caregivers, and healthcare systems in general. The latest drug development pipeline shows that, in 2020, there were 121 compounds in clinical trials for the treatment of AD [10]. The lack of success in AD drug development reveals the complexity of this disease and the current challenges of AD neuropharmacology research. Suggestions to abandon the amyloid hypothesis are increasing and emerging interest is focused on combination therapies [10]. Progress in AD management depends on innovation, the assessment of new candidates, and the implementation of new trial approaches. As in other chronic diseases, such as cardiovascular disease or cancer, a learning phase preceded periods of sequential incremental success leading to meaningful treatments. Therefore, the increase of studies of combinations of new biomarkers and drug targets, combined with novel research in state-of-theart nanocarriers, will pave the future for the more efficient delivery of bioactive molecules. Furthermore, the clinical development of biomarkers of $\mathrm{AD}$ progression to create treatments that are more efficacious will be one of the most ambitious challenges for future clinical practice. In that regard, recent advances on nanomedicine-drug development and novel diagnostic biomarkers could represent a promising alternative in the management of $\mathrm{AD}$, as well as other neurodegenerative diseases.

\section{Abbreviations}

AA: Ascorbic acid; AB: Amyloid-B; AD: Alzheimer's disease; APP: Amyloidprecursor protein; BBB: Blood-brain barrier; CDs: Carbon dots; CSF: Cerebrospinal fluid; CU: Cognitively unimpaired; DALYs: Disability-adjusted life years; DBP: Vitamin D-binding protein; EGCG: Epigallocatechin-3-gallate; EOAD: Early onset AD; EPO: Erythropoietin; FABP3: Heart fatty acid-binding protein 3; FDA: Food and Drug Administration; GAP-43: Growth-associated protein 43; LNPs: Lipid-based nanoparticles; LOAD: Late onset AD; LT: Lutein; MNPs: Metal-based nanoparticles; $\mathrm{MCl}$ : Mild cognitive impairment; ND: Neurodegenerative diseases; NIR: Phenothiazine-based near-infrared; NfL: Neurofilament light; NFTs: Neurofibrillary tangles; Ng: Neurogranin; NLCs: Nanostructured lipid carriers; NPs: Nanoparticles; PCL: Poly- $\varepsilon$-caprolactone; PEI: Polyethyleneimine; PLA: Polylactide; PLGA: Poly(lactide-co-glycolide); PNPs: Polymeric nanoparticles; PS1: Presenilin-1; PS2: Presenilin-2; p-tau: Hyperphosphorylated tau; SLNs: Solid lipid nanoparticles; sTREM2: Soluble ectodomain of TREM2; TREM2: Triggering receptor expressed on myeloid cells 2; t-tau: Total tau; USPIONs: Ultrasmall superparamagnetic iron oxide nanoparticles; VILIP-1: Visinin-like protein 1.

\section{Acknowledgements}

Authors acknowledge the support of the Instituto de Salud Carlos III (ISCIII) Acción Estratégica en Salud, integrated in the Spanish National R+D+I Plan and financed by ISCIII Subdirección General de Evaluación and the Fondo Europeo de Desarrollo Regional (FEDER "Una manera de hacer Europa") grant PI17/01474 awarded to Mercè Boada and grant PI19/00335 awarded to Marta Marquié; Spanish Ministry of Economy and Competitiveness (SAF2017-

84283-R); Biomedical Research Networking Centre in Neurodegenerative
Diseases (CIBERNED, CB06/05/0024); Portuguese Science and Technology Foundation (FCT) for the strategic fund (UIDB/04469/2020) and European Regional Development Funds.

\section{Authors' contributions}

AC: proposal and schematization of the article, bibliography search, content analysis \& selection, writing of original full draft, writing of revision full draft, figure design. PT: content analysis \& selection, writing of original draft section, language correction. ME: bibliography search, content analysis \& selection, writing of original draft section, table edition. JTD: bibliography search, writing of original draft section, language correction. GT: writing of original draft section, writing-review \& editing. ESL: bibliography search, writing of original draft section. MLG: content analysis \& selection, writing-review \& editing. AC: content analysis \& selection, writing-review \& editing. EBS: content proposal, writing-review \& editing, language correction. AR: content proposal, writingreview \& editing, revision of reviewed manuscript. MM: content proposal, manuscript direction, writing-review \& editing, revision of reviewed manuscript. MB: content proposal, manuscript direction, writing-review \& editing. All authors read and approved the final manuscript.

\section{Funding}

Not applicable.

\section{Availability of data and materials}

Not applicable.

\section{Declarations}

Ethics approval and consent to participate

Not applicable.

\section{Consent for publication}

The corresponding author of this manuscript, Dr. Amanda Cano, on behalf of all co-authors (Patric Turowski, Miren Ettcheto, Jason Thomas Duskey, Giovanni Tosi, Elena Sánchez-López, Maria Luisa García, Antonio Camins, Eliana B. Souto, Agustín Ruiz, Marta Marquié and Mercè Boada) declares the consent of publication of this manuscript in the Journal of Nanobiotechnology. All authors have read and approved the submitted final version.

\section{Competing interests}

None of the authors has any conflicts of interest including any financial, personal or other relationships with other people or organizations. All authors have reviewed the contents of the manuscript being submitted and approved its contents.

\section{Author details \\ ${ }^{1}$ Research Center and Memory Clinic, Fundació ACE. Institut Català de Neuro- ciències Aplicades, International University of Catalunya (UIC), C/Marquès de Sentmenat, 57, 08029 Barcelona, Spain. ${ }^{2}$ Biomedical Research Networking Centre in Neurodegenerative Diseases (CIBERNED), Madrid, Spain. ${ }^{3}$ Institute of Nanoscience and Nanotechnology (IN2UB), Barcelona, Spain. ${ }^{4}$ Department of Pharmacy, Pharmaceutical Technology and Physical Chemistry, Faculty of Pharmacy and Food Sciences, University of Barcelona, Barcelona, Spain. ${ }^{5} \mathrm{UCL}$ Institute of Ophthalmology, University College of London, London, UK. ${ }^{6}$ Department of Pharmacology, Toxicology and Therapeutic Chemistry, Faculty of Pharmacy and Food Sciences, University of Barcelona, Barcelona, Spain. ${ }^{7}$ Nanotech Lab, Te.Far.T.I, Department of Life Sciences, University of Modena and Reggio Emilia, Modena, Italy. ${ }^{8}$ Umberto Veronesi Founda- tion, 20121 Milano, Italy. ${ }^{9}$ Department of Pharmaceutical Technology, Faculty of Pharmacy, University of Coimbra, Coimbra, Portugal. ${ }^{10} \mathrm{CEB}$ - Centre of Bio- logical Engineering, University of Minho, Campus de Gualtar, 4710-057 Braga, Portugal.}

Received: 20 January 2021 Accepted: 17 April 2021

Published online: 29 April 2021 


\section{References}

1. Nichols E, Szoeke CEl, Vollset SE, Abbasi N, Abd-Allah F, Abdela J, et al. Global, regional, and national burden of Alzheimer's disease and other dementias, 1990-2016: a systematic analysis for the Global Burden of Disease Study 2016. Lancet Neurol. 2019;18:88-106.

2. Prince PM, Prince $M$, Prince $M$, Karagiannidou M, Prince $M$, Karagiannidou M, et al. World Alzheimer Report 2016 Improving healthcare for people living with dementia. 2016.

3. Cummings JL, Isaacson RS, Schmitt FA, Velting DM. A practical algorithm for managing Alzheimer's disease: what, when, and why? Ann Clin Transl Neurol. 2015;2(3):307-23.

4. Anand R, Dip K, Ali A. Therapeutics of Alzheimer's disease : past, present and future. Neuropharmacology. 2014;76:27-50.

5. Mendez MF, Program BN, Greater VA, Angeles L. Early-onset Alzheimer's disease. Neurol Clin. 2017;35(2):263-81.

6. Hang T, Seelaar H, Melhem S, Rozemuller AJM, Van Swieten JC. Genetic screening in early-onset Alzheimer's disease identified three novel presenilin mutations. Neurobiol Aging. 2020:86:201.e9-201.e14.

7. Ettcheto M, Cano A, Busquets O, Manzine P, Sánchez-López E, CastroTorres $\mathrm{R}$, et al. A metabolic perspective of late onset Alzheimer's disease. Pharmacol Res. 2019;145:104255.

8. Nation DA, Sweeney MD, Montagne A, Sagare AP, D'Orazio LM, Maricarmen P, Sepehrband F. Blood-brain barrier breakdown is an early biomarker of human cognitive dysfunction. Nat Med. 2019:25:270-6.

9. Nutma E, Amor S, Peferoen LAN. Astrocyte and oligodendrocyte crosstalk in the central nervous system. Cells. 2020;9:600.

10. Cummings J, Zhong K, Lee G, Ritter A, Sabbagh M. Alzheimer's disease drug development pipeline: 2020. Alzheimer's Dement. 2020;6:e12050.

11. Mulvihill JJ, Cunnane EM, Ross AM, Duskey JT, Tosi G, Grabrucker AM Drug delivery across the blood-brain barrier: recent advances in the use of nanocarriers. Nanomedicine (London). 2020:5(2):205-14.

12. Duskey J, Belletti D, Pederzoli F, Vandelli M, Forni F, Ruozi B, et al. Current strategies for the delivery of therapeutic proteins and enzymes to treat brain disorders. Int Rev Neurobiol. 2017;137:1-28.

13. Wilczewska AZ, Niemirowicz K, Markiewicz KH, Car H. Nanoparticles as drug delivery systems. Pharmacol Rep. 2012;64(5):1020-37.

14. Duskey JT, Ottonelli I, Da Ros F, Vilella A, Zoli M, Kovachka S, et al. Novel peptide-conjugated nanomedicines for brain targeting: in vivo evidence. Nanomed Nanotechnol Biol Med. 2020;28:102226.

15. Hippius H. The discovery of Alzheimer's disease. Dialogues Clin Neurosci. 2003;5(1):101-8.

16. Dubois B, Feldman HH, Cummings JL, Dekosky ST, Barberger-Gateau P, Delacourte A, Frisoni G. Revising the definition of Alzheimer's disease: a new lexicon. Lancet Neurol. 2010;9(11):1118-27.

17. Alegret M, Muñoz N, Roberto N, Rentz DM, Valero S, Gil S, et al. A computerized version of the Short Form of the Face-Name Associative Memory Exam (FACEmemory ${ }^{\circledR}$ ) for the early detection of Alzheimer's disease. Alzheimers Res Ther. 2020;12(1):25

18. Khoury R, Ghossoub E. Diagnostic biomarkers of Alzheimer's disease: a state-of-the-art review. Biomark Neuropsychiatr. 2019;1:100005.

19. Jack CR Jr, Bennett DA, Blennow K, Carrillo MC, Dunn B, Haeberlein S, Holtzman DM, et al. NIA-AA research framework: toward a biological definition of Alzheimer's disease. Alzheimers Dement. 2018;14(4):535-62.

20. El KN, Said N, Slassi I, Moutawakil E, Nadifi S. Biomarkers for Alzheimer Disease: classical and novel candidates 'review. Neuroscience. 2018;370:181-90

21. Canevelli M, Bacigalupo I, Gervasi G, Lacorte E, Massari M, Mayer F, et al. Methodological Issues in the clinical validation of biomarkers for Alzheimer's Disease: the paradigmatic example of CSF. Front Aging Neurosci. 2019;11:282.

22. Rossini PM, lorio RD, Vecchio F, Anfossi M, Babiloni C, Bozzali M, et al. Early diagnosis of Alzheimer's disease : the role of biomarkers including advanced EEG signal analysis. Report from the IFCN-sponsored panel of experts. Cli Neurophysiol. 2020;131(6):1287-310.

23. Blennow K, Dubois B, Fagan A, Lewczuk P, de Leon M, Hampel H. Clinical utility of cerebrospinal fluid biomarkers in the diagnosis of early Alzheimer's disease. Alzheimer's Dementia. 2015;11(1):58-69.

24. Whelan CD, Mattsson N, Nagle MW, Vijayaraghavan S, Hyde C, Janelidze $S$, et al. Multiplex proteomics identifies novel CSF and plasma biomarkers of early Alzheimer 's disease. Acta Neuropathol Commun. 2019;7(1):169.

25. Trombetta BA, Carlyle BC, Koenig AM, Shaw LM, Trojanowski Q, Wolk $D A$, et al. The technical reliability and biotemporal stability of cerebrospinal fluid biomarkers for profiling multiple pathophysiologies in Alzheimer's disease. PLoS ONE. 2018;13(3):e0193707.

26. Olsson B, Lautner R, Andreasson U, Öhrfelt A, Portelius E, Bjerke M, et al. CSF and blood biomarkers for the diagnosis of Alzheimer's disease: a systematic review and meta-analysis. Lancet Neurol. 2016;15(7):673-84.

27. Park SA, Han S, Kim CE. New fluid biomarkers tracking non-amyloid$\beta$ and non-tau pathology in Alzheimer's disease. Exp Mol Med. 2020;52:556-68.

28. Awasthi M, Singh S, Pandey VP, Dwivedi UN. Alzheimer $\hat{a} €^{\mathrm{TM}}$ S disease: an overview of amyloid beta dependent pathogenesis and its therapeutic implications along with in silico approaches emphasizing the role of natural products. J Neurol Sci. 2016:361:256-71.

29. Milà-Alomà M, Suárez-calvet M, Molinuevo JL. Latest advances in cerebrospinal fluid and blood biomarkers of Alzheimer 's disease. Ther Adv Neurol Disord. 2019;12:1-23.

30. Hrubešová K, Fousková M, Habartová L, Fišar Z, Jirák R. Search for biomarkers of Alzheimer' s disease: recent insights, current challenges and future prospects. Clin Biochem. 2019;72:39-51.

31. Lewczuk P, Matzen A, Blennow K, Parnetti L, Molinuevo JL, Eusebi P, et al Cerebrospinal fluid $A \beta 42 / 40$ corresponds better than $A \beta 42$ to amyloid PET in Alzheimer's Disease. J Alzheimers Dis. 2017:55(2):813-22.

32. Janelidze S, Zetterberg $H$, Mattsson N, Palmqvist S, Vanderstichele $H$, Lindberg $O$, et al. CSF $A \beta 42 / A \beta 40$ and $A \beta 42 / A \beta 38$ ratios: better diagnostic markers of Alzheimer disease. Ann Clin Transl Neurol. 2016;3(3):154-65.

33. Pérez-Grijalba V, Romero J, Pesini P, Sarasa L, Monleón I, San-José I, et al. Plasma A $42 / 40$ ratio detects early stages of Alzheimer's disease and correlates with CSF and neuroimaging biomarkers in the AB255 study. J Prev Alzheimers Dis. 2019;6(1):34-41.

34. Pérez-Grijalba V, Arbizu J, Romero J, Prieto E, Pesini P, Sarasa L, et al. Plasma A $342 / 40$ ratio alone or combined with FDG-PET can accurately predict amyloid-PET positivity: a cross-sectional analysis from the AB255 Study. Alzheimers Res Ther. 2019;11(1):96.

35. Zetterberg H. Blood-based biomarkers for Alzheimer's disease-an update. J Neurosci Methods. 2019;319:2-6.

36. Huynh RA, Mohan C, Huynh RA, Mohan C. Alzheimer's Disease: biomarkers in the genome, blood, and cerebrospinal fluid. Front Neurol. 2017:8:102.

37. Schuster J, Funke SA. Methods for the specific detection and quantitation of amyloid- $\beta$ oligomers in cerebrospinal fluid. J Alzheimers Dis. 2016;53(1):53-67.

38. Alzforum. Plasma A $\beta$ test wins approval —are $p$-tau tests far behind?. 2020. https://www.alzforum.org/news/research-news/plasma-av-testwins-approval-are-p-tau-tests-far-behind.

39. Hampel H, Vassar R, De Strooper B, Hardy J, Willem M, Singh N, et al. The $\beta$-Secretase BACE1 in Alzheimer's Disease. Biological Psychiatry. 2020;In press.

40. Mulder SD, van der Flier WM, Verheijen JH, Mulder C, Scheltens P, Blankenstein MA, et al. BACE1 activity in cerebrospinal fluid and its relation to markers of AD pathology. J Alzheimers Dis. 2010;20(1):253-60.

41. Ewers $M$, Cheng $X$, Zhong Z, Nural HF, Walsh C, Meindl T, et al. Increased CSF-BACE1 activity associated with decreased hippocampus volume in Alzheimer's disease. J Alzheimers Dis. 2011;25(2):373-81.

42. Perneczky R, Alexopoulos P. Cerebrospinal fluid BACE1 activity and markers of amyloid precursor protein metabolism and axonal degeneration in Alzheimer's disease. Alzheimers Dement. 2014;10:S425-S429. e1.

43. Savage MJ, Holder DJ, Wu G, Kaplow J, Siuciak JA, Potter WZ. Soluble BACE- 1 activity and SA PPP $\beta$ Concentrations in Alzheimer's Disease and age-matched healthy control cerebrospinal fluid from the Alzheimer's Disease neuroimaging initiative-1 baseline cohort. J Alzheimers Dis. 2015:46(2):431-40.

44. Shen Y, Wang H, Sun Q, Yao H, Keegan AP, Mullan M, et al. Increased plasma BACE1 may predict conversion to Alzheimer's Disease dementia in individuals with mild cognitive impairment. Biol Psychiatry. 2018:83(5):447-55. 
45. Grundke-lqbal I, lqbal K, Tung Y, Quinlan M, Wisniewski H, Binder L. Abnormal phosphorylation of the microtubule-associated protein tau (tau) in Alzheimer cytoskeletal pathology. Proc Natl Acad Sci U S A. 1986;83(13):4913-7.

46. Arendt T, Stieler JT, Holzer M. Tau and tauopathies. Brain Res Bull. 2016;126:238-92.

47. Schöll M, Maass A, Mattsson N, Ashton NJ, Blennow K, Zetterberg H, et al. Biomarkers for tau pathology. Mol Cell Neurosci. 2019;97:18-33.

48. Wattmo C, Blennow K, Hansson O. Cerebro-spinal fluid biomarker levels: phosphorylated tau ( $\mathrm{T}$ ) and total tau (N) as markers for rate of progression in Alzheimer's disease. BMC Neurol. 2020;20:10.

49. Aschenbrenner AJ, Gordon BA, Benzinger TLS, Morris JC, Hassensta JJ. Influence of tau PET, amyloid PET, and hippocampal volume on cognition in Alzheimer disease. Neurology. 2018;91(9):e859-66.

50. Lleó A, Alcolea D, Martínez-Lage P, Scheltens P, Parnetti L, Poirier J, et al. Longitudinal cerebrospinal fluid biomarker trajectories along the Alzheimer's disease continuum in the BIOMARKAPD study. Alzheimers Dement. 2019;151(6):742-53.

51. Mielke MM, Hagen CE, Xu J, Chai X, Vemuri P, Lowe VJ, et al. Plasma phospho-tau181 increases with Alzheimer's disease clinical severity and is associated with tau- and amyloid-positron emission tomography. Alzheimers Dement. 2018;14(8):989-97.

52. Shekhar S, Kumar R, Rai N, Kumar V, Singh K, Upadhyay AD, et al. Estimation of tau and phosphorylated tau181 in serum of Alzheimer's Disease and mild cognitive impairment patients. PLoS ONE. 2016;11(7):e0159099.

53. Yang C-C, Chiu M-J, Chen T-F, Chang H-L, Liu B-H, Yang S-Y. Assay of plasma phosphorylated tau protein (Threonine 181) and total tau protein in early-stage Alzheimer's Disease. J Alzheimers Dis. 2018;61(4):1323-32.

54. Palmavist $\mathrm{S}$, Janelidze $\mathrm{S}$, Quiroz $\mathrm{YT}$, Zetterberg H, Lopera F, Stomrud $\mathrm{E}$, et al. Discriminative accuracy of plasma phospho-tau217 for Alzheimer Disease vs other neurodegenerative disorders. JAMA. 2020;324(8):772-81.

55. Barthélemy NR, Horie K, Sato C, Bateman RJ. Blood plasma phosphorylated-tau isoforms track CNS change in Alzheimer's disease. J Exp Med. 2020;217(11):e20200861.

56. Suárez-Calvet M, Karikari T, Ashton N, Lantero Rodríquez J, Milà-Alomà M, Gispert J, et al. Novel tau biomarkers phosphorylated at T181, T217 or T231 rise in the initial stages of the preclinical Alzheimer's continuum when only subtle changes in $A \beta$ pathology are detected. EMBO Mol Med. 2020;10:e12921.

57. Mattsson-Carlgren N, Janelidze S, Palmqvist S, Cullen N, Svenningsson A, Strandberg O, et al. Longitudinal plasma p-tau217 is increased in early stages of Alzheimer's disease. Brain. 2020;17:3234.

58. Bos I, Vos S, Verhey F, Scheltens P, Teunissen C, Engelborghs S, Sleegers K. Cerebrospinal fluid biomarkers of neurodegeneration, synaptic integrity, and astroglial activation across the clinical Alzheimer's disease spectrum. Alzheimers Dement. 2019;15(5):644-54.

59. Skillbäck T, Farahmand B, Bartlett JW, Rosén C, Mattsson N, Nägga K, et al. CSF neurofilament light differs in neurodegenerative diseases and predicts severity and survival. Neurology. 2014;83(21):1945-53.

60. Mattsson N, Cullen NC, Andreasson U, Zetterberg H, Blennow K. Association between longitudinal plasma neurofilament light and neurodegeneration in patients with Alzheimer Disease. JAMA Neurol. 2019;76(7):791-9.

61. Weston PSJ, Poole T, O'Connor A, Heslegrave A, Ryan NS, Liang Y, et al. Longitudinal measurement of serum neurofilament light in presymptomatic familial Alzheimer's disease. Alzheimers Res Ther. 2019;11(1):19.

62. Raket LL, Kühnel L, Schmidt E, Blennow K, Zetterberg H, MattssonCarlgren N. Utility of plasma neurofilament light and total tau for clinical trials in Alzheimer's disease. Alzheimers Dement (Amst). 2020;12(1):e12099.

63. Groblewska M, Muszyński P,Wojtulewska-Supron A, Kulczyńska-Przybik A, Mroczko B. The role of visinin-like protein-1 in the pathophysiology of Alzheimer's Disease. J Alzheimers Dis. 2015;47(1):17-32.

64. Zhang H, Pin Ng K, Therriault J, Kang MS, Pascoal TA, Rosa-Neto P, et al. Cerebrospinal fluid phosphorylated tau, visinin-like protein-1, and chitinase-3-like protein 1 in mild cognitive impairment and Alzheimer's disease. Transl Neurodegener. 2018;7:23.
65. Regen F, Hellmann-Regen J, Costantini E, Reale M. Neuroinflammation and Alzheimer's Disease: implications for microglial activation. Curr Alzheimer Res. 2017;14(11):1140-448.

66. Calsolaro V, Edison P. Neuroinflammation in Alzheimer's disease: current evidence and future directions. Alzheimer's Dementia. 2016;12(6):719-32.

67. Mizuno T. The biphasic role of microglia in Alzheimer's disease. Int J Alzheimer's Dis. 2012;2012(737846):1-9.

68. Condello C, Yuan P, Grutzendler J. Microglia-mediated neuroprotection, TREM2, and Alzheimer's Disease: evidence from optical imaging. Biol Psychiat. 2018;83(4):377-87.

69. Sjögren $M$, Blomberg $M$, Jonsson $M$, Wahlund L, Edman $A$, Lind $K$, et al. Neurofilament protein in cerebrospinal fluid: a marker of white matter changes. J Neurosci Res. 2001;66(3):510-6.

70. Parbo P, Madsen LS, Ismail R, Zetterberg H, Blennow K, Eskildsen SF, et al. Low plasma neurofilament light levels associated with raised cortical microglial activation suggest inflammation acts to protect prodromal Alzheimer 's disease. Alzheimer's Res Therapy. 2020;12(3):1-7.

71. Khalil M, Pirpamer L, Hofer E, Voortman MM, Barro C, Leppert D, et al. Serum neurofilament light levels in normal aging and their association with morphologic brain changes. Nat Commun. 2020;11:812. https://doi.org/10.1038/s41467-020-14612-6.

72. Baldacci F, Lista S, Cavedo E, Bonuccelli U, Hampel H. Diagnostic function of the neuroinflammatory biomarker YKL-40 in Alzheimer's disease and other neurodegenerative diseases. Expert Rev Proteomics. 2017;14(4):285-99.

73. Mendsaikhan A, Tooyama I, Walker DG. Microglial progranulin: involvement in Alzheimer's Disease and neurodegenerative diseases. Cells. 2019;8(3):230.

74. Ahmed Z, Mackenzie IR, Hutton ML, Dickson DW. Progranulin in frontotemporal lobar degeneration and neuroinflammation. J Neuroinflammation. 2007:4(7):1-13.

75. Suárez-Calvet M, Capell A, Araque Caballero MÁ, Morenas-Rodríguez $\mathrm{E}$, Fellerer $\mathrm{K}$, Franzmeier $\mathrm{N}$, et al. CSF progranulin increases in the course of Alzheimer's disease and is associated with sTREM2, neurodegeneration and cognitive decline. EMBO Mol Med. 2018;10(12):e9712.

76. Wilke C, Gillardon F, Deuschle C, Dubois E, Hobert MA, vom Hagen $\mathrm{MJ}$, et al. Serum levels of progranulin do not reflect cerebrospinal fluid levels in neurodegenerative disease. Curr Alzheimer Res. 2016;13(6):654-62.

77. Zhou Y, Ulland TK, Colonna M. TREM2-dependent effects on microglia in Alzheimer's Disease. Front Aging Neurosci. 2018;10:202.

78. Jonsson T, Stefansson H, Steinberg S, Jonsdottir I, Jonsson PV, Snaedal J, et al. Variant of TREM2 associated with the risk of Alzheimer's disease. N Engl J Med. 2013;368(2):107-16.

79. Chen S, Peng J, Sherchan P, Ma Y, Xiang S, Yan F, et al. TREM2 activation attenuates neuroinflammation and neuronal apoptosis via PI3K/Akt pathway after intracerebral hemorrhage in mice. J Neuroinflammation. 2020;17:168.

80. Zhong L, Xu Y, Zhuo R, Wang T, Wang K, Huang R, et al. Soluble TREM2 ameliorates pathological phenotypes by modulating microglial functions in an Alzheimer's disease model. Nat Commun. 2019;10(1):1365.

81. Ewers M, Biechele G, Suárez-Calvet M, Sacher C, Blume T, MorenasRodriguez E, et al. Higher CSF sTREM2 and microglia activation are associated with slower rates of beta-amyloid accumulation. EMBO Mol Med. 2020;12(9):e12308.

82. Lista S, Hampel H. Synaptic degeneration and neurogranin in the pathophysiology of Alzheimer's disease. Expert Rev Neurother. 2017;17(1):47-57.

83. Overk CR, Masliah E. Pathogenesis of synaptic degeneration in Alzheimer's disease and Lewy Body disease. Biochem Pharmacol. 2014:88(4):508-16.

84. Cheetham JE, Martzen MR, Kazee AM, Coleman PD. Gap-43 message levels in anterior cerebellum in Alzheimer's disease. Brain Res Mol Brain Res. 1996;36(1):145-51.

85. Sandelius Å, Portelius E, Källén Å, Zetterberg H, Rot U, Olsson B, et al. Elevated CSF GAP-43 is Alzheimer's disease specific and associated with tau and amyloid pathology. Alzheimers Dement. 2019;15(1):55-64. 
86. Zhang H, Therriault J, Kang MS, Pin Ng K, Pascoal TA, Rosa-Neto P, et al. Cerebrospinal fluid synaptosomal-associated protein 25 is a key player in synaptic degeneration in mild cognitive impairment and Alzheimer's disease. Alzheimers Res Ther. 2018;10(1):80

87. Wang S, Zhang J, Pan T. APOE $\varepsilon 4$ is associated with higher levels of CSF SNAP-25 in prodromal Alzheimer's disease. Neurosci Lett. 2018;685:109-13.

88. Agliardi C, Guerini FR, Zanzottera M, Bianchi A, Nemni R, Clerici M. SNAP-25 in serum is carried by exosomes of neuronal origin and is a potential biomarker of Alzheimer's Disease. Mol Neurobiol. 2019;56(8):5792-8.

89. Davidsson P, Jahn R, Bergquist J, Ekman R, Blennow K. Synaptotagmin, a synaptic vesicle protein, is present in human cerebrospinal fluid: a new biochemical marker for synaptic pathology in Alzheimer disease? Mol Chem Neuropathol. 1996;27(2):195-210.

90. Öhrfelt A, Brinkmalm A, Dumurgier J, Brinkmalm G, Hansson O, Zetterberg $\mathrm{H}$, et al. The pre-synaptic vesicle protein synaptotagmin is a novel biomarker for Alzheimer's disease. Alzheimers Res Ther. 2016;8(1):41.

91. Tarawneh R, D'Angelo G, Crimmins D, Herries E, Griest T, Fagan AM, et al. Diagnostic and prognostic utility of the synaptic marker neurogranin in Alzheimer Disease. JAMA Neurol. 2016;73(5):561-71.

92. Dhiman K, Blennow K, Zetterberg H, Martins RN, Gupta VB. Cerebrospinal fluid biomarkers for understanding multiple aspects of Alzheimer's disease pathogenesis. Cell Mol Life Sci. 2019;76(10):1833-63.

93. Kirsebom B-E, Nordengen K, Selnes P, Waterloo K, Bøen Torsetnes S, Gísladóttir B, et al. Cerebrospinal fluid neurogranin/ $\beta$-site APP-cleaving enzyme 1 predicts cognitive decline in preclinical Alzheimer's disease. Alzheimers Dement (NY). 2018;4:617-27.

94. Di Marco LY, Venneri A, Farkas E, Evans PC, Marzo A, Frangi AF. Vascular dysfunction in the pathogenesis of Alzheimer's disease-a review of endothelium-mediated mechanisms and ensuing vicious circles. Neurobiol Dis. 2015;82:593-606.

95. Tibbling $G$, Link H, Ohman S. Principles of albumin and Ig G analyses in neurological disorders. I. Establishment of reference values. Scand J Clin Lab Invest. 1997;37(5):385-90.

96. Wong MW, Braidy N, Poljak A, Pickford R, Thambisetty M, Sachdev PS. Dysregulation of lipids in Alzheimer's disease and their role as potential biomarkers. Alzheimers Dement. 2017;13(7):810-27.

97. Belloy ME, Napolioni V, Greicius MD. A quarter century of APOE and Alzheimer's Disease: progress to date and the path forward. Neuron. 2019;101(5):820-38.

98. Ries M, Sastre M. Mechanisms of A $\beta$ clearance and degradation by glial cells. Front Aging Neurosci. 2016;8:160.

99. Heywood WE, Galimberti D, Bliss E, Sirka E, Paterson RW, Magdalinou NK, et al. Identification of novel CSF biomarkers for neurodegeneration and their validation by a high-throughput multiplexed targeted proteomic assay. Mol Neurodegener. 2015;10:64

100. Wang L, Yin Y, Liu X, Shen P, Zheng Y, Lan X, et al. Current understanding of metal ions in the pathogenesis of Alzheimer's disease. Transl Neurodegen. 2020;9(10):1-13.

101. Wang S, Mims PN, Roman RJ, Fan F. Is beta-amyloid accumulation a cause or consequence of Alzheimer's Disease? J Alzheimers Parkinsonism Dement. 2016;1 (2):1-8.

102. Ballard C, Gauthier S, Corbett A, Brayne C, Aarsland D, Jones E. Alzheimer's disease. Lancet. 2011;377(9770):1019-31.

103. Mroczko B, Groblewska M, Litman-zawadzka A, Kornhuber J, Lewczuk P. Cellular receptors of amyloid $\beta$ oligomers (AßOs) in Alzheimer's Disease. Int J Mol Sci. 2018;19:1884

104. Smith A, Giunta B, Bickford PC, Fountain M, Tan J, Shytle RD. Nanolipidic particles improve the bioavailability and a-secretase inducing ability of epigallocatechin-3-gallate (EGCG) for the treatment of Alzheimer's disease. Int J Pharm. 2010;389(1-2):207-12.

105. de la Torre R. Prevention of cognitive decline in ApoE4 Carriers with subjective cognitive decline after EGCG and a multimodal intervention ((PENSA)). ClinicalTrials.gov. 2021. https://clinicaltrials.gov/ct2/show/ NCT03978052.

106. Marcade M, Bourdin J, Loiseau N, Peillon H, Rayer A, Drouin D, et al. Etazolate, a neuroprotective drug linking GABA(A) receptor pharmacology to amyloid precursor protein processing. J Neurochemistry. 2008; 106:392-404.
107. Li Z. New APOE-related therapeutic options for Alzheimer's disease. AIP Conf Proc. 2019;2058:20002.

108. Kanekiyo T, Bu G. The low-density lipoprotein receptor-related protein 1 and amyloid- $\beta$ clearance in Alzheimer's disease. Front Aging Neurosci. 2014;6:1-12.

109. Kim DE, Priefer R. Therapeutic potential of direct clearance of the amyloid- $\beta$ in Alzheimer's Disease. Brain Sci. 2020;10:93.

110. Sevigny J, Chiao P, Bussière T, Weinreb PH, Williams L, Maier M, et al. Addendum: the antibody aducanumab reduces $\mathrm{A} \beta$ plaques in Alzheimer's disease. Nature. 2017;546(7659):564.

111. Alzforum. aducanumab still needs to prove itself, researchers say. 2020. https://www.alzforum.org/news/research-news/aducanumabstill-needs-prove-itself-researchers-say.

112. Boada M, Ramos-Fernández E, Guivernau B, Muñoz F, Costa M, Ortiz $A$, et al. Treatment of Alzheimer disease using combination therapy with plasma exchange and haemapheresis with albumin and intravenous immunoglobulin: Rationale and treatment approach of the AMBAR (Alzheimer Management By Albumin Replacement) study. Neurologia. 2016;31(7):473-81.

113. Boada M, López O, Núñez L, Szczepiorkowski ZM, Torres M, Grifols $C$, et al. Plasma exchange for Alzheimer's disease Management by Albumin Replacement (AMBAR) trial: study design and progress. Alzheimers Dement (N Y). 2019;5:61-9.

114. Boada M, López OL, Olazarán J, Núñez L, Pfeffer M, Paricio M, et al. A randomized, controlled clinical trial of plasma exchange with albumin replacement for Alzheimer's disease: primary results of the AMBAR Study. Alzheimer's Dement. 2020;16:1412-25.

115. Chong FP, Ng KY, Koh RY, Moi CS. Tau proteins and tauopathies in Alzheimer's Disease. Cell Mol Neurobiol Vol. 2018;38:965-80.

116. Rajmohan R, Reddy PH. Amyloid beta and phosphorylated tau accumulations cause abnormalities at synapses of Alzheimer's Disease neurons. J Alzheimers Dis. 2018;57(4):975-99.

117. Avila J, León-Espinosa G, García E, García-Escudero V, Hernández F, DeFelipe J. Tau phosphorylation by GSK3 in different conditions. Int J Alzheimer's Dis. 2012;2012:578373.

118. Hampel H, Mesulam M, Cuello AC, Farlow MR, Giacobini E, Grossberg $\mathrm{GT}$, et al. The cholinergic system in the pathophysiology and treatment of Alzheimer's disease. Brain. 2018;141:1917-33.

119. Pittel Z, Heldman E, Barg J, Haring R, Fisher A. Muscarinic control of amyloid precursor protein secretion in rat cerebral cortex and cerebellum. Brain Res. 1996;742(1-2):299-304.

120. Gandía L, Álvarez R, De Pascual R, Rojo J, Tapia L. Anticholinesterases in the treatment of Alzheimer's disease. Rev Neurol. 2006;42(8):471-7.

121. López-Álvarez J, Sevilla-Llewellyn-Jones J, Agüera-Ortiz L. Anticholinergic drugs in geriatric psychopharmacology. Front Neurosci. 2019:13:1309.

122. Suzuki M, Nelson A, Eickstaedt J, Wallace K, Wright L, Svendsen CN. Glutamate enhances proliferation and neurogenesis in human neural progenitor cell cultures derived from the fetal cortex. Eur I Neurosci. 2006;24(3):645-53.

123. Danysz W, Parsons C. Alzheimer's disease, $\beta$-amyloid, glutamate, NMDA receptors and memantine-searching for the connections. Br J Pharmacol. 2012;167(2):324-52.

124. Huang WJ, Zhang X, Chen W. Role of oxidative stress in Alzheimer's disease. Biomed Rep. 2016:4:519-22.

125. Nunomura A, Perry G, Aliev G, Hirai K, Takeda A, Balraj EK, et al. Oxidative damage is the earliest event in Alzheimer disease. J Neuropathol Exp Neurol. 2009;60(8):759-67.

126. Essa MM, Akbar M, Gilles G. The benefits of natural products for neurodegenerative diseases. 2016. pp. 107-123.

127. Hemonnot A, Hua J, Ulmann L, Hirbec H. Microglia in Alzheimer Disease: well-known targets and new opportunities. Front Aging Neurosci. 2019;11:1-20.

128. Arranz AM, De SB. The role of astroglia in Alzheimer's disease: pathophysiology and clinical implications. Lancet Neurol. 2019:18(4):406-14

129. Bronzuoli M, lacomino A, Steardo L, Scuderi C. Targeting neuroinflammation in Alzheimer's disease. J Inflamm Res. 2016;9:199-208.

130. Ali MM, Ghouri RG, Ans AH, Akbar A, Toheed A. Recommendations for anti-inflammatory treatments in Alzheimer's Disease: a comprehensive review of the literature. Cureus. 2019;11(5):e4620. 
131. Onyango IG, Dennis J, Khan SM, Biotechnology G, St BF. Mitochondrial dysfunction in Alzheimer's Disease and the rationale for bioenergetics based therapies. Aging Dis. 2016;7(2):201-14

132. Anand R, Gill K, Mahdi A. Therapeutics of Alzheimer's disease: past, present and future. Neuropharmacology. 2014;76:27-50.

133. Maczurek A, Hager K, Kenklies M, Sharman M, Martins R, Engel J, et al. Lipoic acid as an anti-inflammatory and neuroprotective treatment for Alzheimer's disease. Adv Drug Deliv Rev. 2008;60:1463-70.

134. Bachurin S, Bukatina E, Lermontova N, Tkachenko S, Afanasiev A, Grigo-

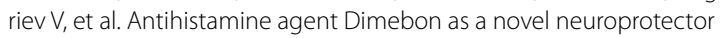
and a cognition enhancer. Ann N Y Acad Sci. 2001;939:425-35.

135. Woong Lim J, Lee J, Pae N. Mitochondrial dysfunction and Alzheimer's disease: prospects for therapeutic intervention. BMB Rep. 2020;53(1):47-55

136. Craft S. The role of metabolic disorders in Alzheimer's Disease and vascular dementia: two roads converged? Arch Neurol. 2009;66(3):300-5.

137. Chu C-S, Tseng P-T, Stubbs B, Chen T-Y, Tang C-H, Li D-J, et al. Use of statins and the risk of dementia and mild cognitive impairment: a systematic review and meta-analysis. Sci Rep. 2018;8:5804.

138. Bendlin BB. Antidiabetic therapies and Alzheimer disease. Dialogues Clin Neurosci. 2019;21(1):83-91.

139. Yun YH, Lee BK, Park K, Lafayette W. Controlled drug delivery: historical perspective for the next generation. J Control Release. 2015;219:2-7.

140. Yun Y, Lee B, Park K. Controlled drug delivery systems: the next 30 years. Front Chem Sci Eng. 2014;8(3):276-9.

141. Bassyouni F, Elhalwany N. Advances and new technologies applied in controlled drug delivery system. Res Chem Intermed. 2015;41:2165-200.

142. Benoit DSW, Overby CT, Jr KRS, Ackun-farmmer MA. 2.5.12 - Drug Delivery Systems. Fourth Edi. Biomaterials Science. Elsevier Inc.; 2020. pp. 1237-1259.e3. https://doi.org/10.1016/B978-0-12-816137-1.00079-9

143. Derakhshankhah H, Sajadimajd S, Jafari S, Izadi Z, Sarvari S, Sharifi M, et al. Novel therapeutic strategies for Alzheimer's disease: implications from cell-based therapy and nanotherapy. Nanomed Nanotechnol Biol Med. 2020;24:102149.

144. Kouyoumdjian H, Zhu DC, El-Dakdouki MH, Lorenz K, Chen J, Li W, et al. Glyconanoparticle aided detection of $\beta$-Amyloid by Magnetic resonance imaging and attenuation of $\beta$-Amyloid Induced Cytotoxicity. ACS Chem Neurosci. 2013;4(4):575-84.

145. Viola KL, Sbarboro J, Sureka R, De M, Bicca MA, Wang J, et al. Towards non-invasive diagnostic imaging of early-stage Alzheimer's disease. Nat Nanotechnol. 2015;10(1):91-8.

146. Tanifum EA, Ghaghada K, Vollert C, Head E, Eriksen JL, Annapragada A. A novel liposomal nanoparticle for the imaging of amyloid plaque by magnetic resonance imaging. J Alzheimers Dis. 2016;52(2):731-45.

147. Gavhanea YN, Yadav AV. Loss of orally administered drugs in Gl tract. Saudi Pharm J. 2012;20(4):331-44.

148. Hoosain FG, Choonara YE, Tomar LK, Kumar P, Tyagi C, du Toit LC, et al. Bypassing P-glycoprotein drug efflux mechanisms: possible applications in pharmacoresistant schizophrenia therapy. Biomed Res Int. 2015;2015:484963.

149. Lennernäs $H$, Abrahamsson B. The use of biopharmaceutic classification of drugs in drug discovery and development: current status and future extension. J Pharm Pharmacol. 2005;57(3):273-85.

150. Cano A, Sánchez-López E, Ettcheto M, López-Machado A, Espina M Souto EB, et al. Current advances in the development of novel polymeric nanoparticles for the treatment of neurodegenerative diseases. Nanomedicine (London). 2020;15(12):1239-61.

151. Alvarez-Erviti L, Seow Y, Yin H, Betts C, Lakhal S, Wood M. Delivery of siRNA to the mouse brain by systemic injection of targeted exosomes. Nat Biotechnol. 2011;29:341-5.

152. Tajes M, Ramos-fernández E, Weng-jiang X, Bosch-morató M, Guivernau B, Eraso-pichot A, et al. The blood-brain barrier: structure, function and therapeutic approaches to cross it. Mol Membr Biol. 2014;31(5):152-67.

153. Gordillo-galeano A, Mora-huertas CE. Solid lipid nanoparticles and nanostructured lipid carriers: a review emphasizing on particle structure and drug release. Eur J Pharm Biopharm. 2018;133:285-308.

154. Cano A, Espina M, García M. Recent advances on anti-tumor agentsloaded polymeric and lipid based nano-carriers for the treatment of brain cancer. Curr Pharm Des. 2020;26(12):1316-30.
155. Campani V, Giarra S, De RG. Lipid-based core-shell nanoparticles: evolution and potentialities in drug delivery. OpenNano. 2018;3:5-17.

156. Kraft J, Freeling J, Wang Z, Ho R. Emerging research and clinical development trends of liposome and lipid nanoparticle drug delivery systems. J Pharm Sci. 2014;103(1):29-52.

157. Almuhayawi MS, Ramadan WS, Harakeh S, Al SK, Bharali DJ, Mousa SA et al. The potential role of pomegranate and its nano-formulations on cerebral neurons in aluminum chloride induced Alzheimer rat model. Saudi J Biol Sci. 2020;27(7):1710-6.

158. Giacomeli R, Cristina J, Bem R, Peterini S, Ricardo C, Elisa S. Neuroprotective effects of curcumin lipid-core nanocapsules in a model Alzheimer's disease induced by $\beta$-amyloid 1-42 peptide in aged female mice. Brain Res. 2019;1721:146325.

159. Gupta SC, Patchva S, Aggarwal BB. Therapeutic roles of curcumin: lessons learned from clinical trials. AAPS J. 2013;15(1):195-218.

160. Willson J. Transferrin'across the blood-brain barrier. Nat Rev Drug Discov. 2020;19:444

161. Pinheiro RGR, Granja A, Loureiro JA, Pereira MC, Pinheiro M, Neves AR, et al. Quercetin lipid nanoparticles functionalized with transferrin for Alzheimer's disease. Eur J Pharm Sci. 2020;148:105314.

162. Kamatou GPP, Viljoen AM. A review of the application and pharmacological properties of a-Bisabolol and a-Bisabolol-rich oils. JAOCS. 2010;87(1):1-7.

163. Sathya S, Shanmuganathan B, Manirathinam G, Ruckmani K, Pandima K. a -Bisabolol loaded solid lipid nanoparticles attenuates $A \beta$ aggregation and protects Neuro-2a cells from A $\beta$ induced neurotoxicity. J Mol Liq. 2018:264:431-41.

164. Sathya S, Shanmuganathan B, Devi KP. Deciphering the antiapoptotic potential of a -bisabolol loaded solid lipid nanoparticles against $A \beta$ induced neurotoxicity in Neuro-2a cells. Colloids Surf B. 2020:190:110948.

165. Dara T, Vatanara A, Sharifzadeh M, Khani S. Improvement of memory deficits in the rat model of Alzheimer's disease by erythropoietin-loaded solid lipid nanoparticles. Neurobiol Learn Mem. 2019;166:107082

166. Punnonen J, Miller JL, Collier TJ, Spencer JR. Agonists of the tissue-protective erythropoietin receptor in the treatment of Parkinson's disease. Curr Top Med Chem. 2015;15(10):955-69.

167. Cevik B, Solmaz V, Yigitturk G, Cavusoğlu T, Gonul P, Erbas O. Neuroprotective effects of erythropoietin on Alzheimer's dementia model in rats. Adv Clin Exp Med. 2017;26(1):23-9.

168. Kumari A, Yadav SK, Yadav SC. Biodegradable polymeric nanoparticles based drug delivery systems. Colloids Surf B. 2010;75:1-18.

169. Reis CP, Neufeld RJ. Nanoencapsulation. I. Methods for preparation of drug-loaded polymeric nanoparticles. Nanomed Nanotechnol Biol Med. 2006;2:8-21

170. El-say KM, El-sawy HS. Polymeric nanoparticles: Promising platform for drug delivery. Int J Pharm. 2017;528(1-2):675-91.

171. Liu S, Qiao S, Li L, Qi G, Lin Y. Surface charge-conversion polymeric nanoparticles for photodynamic treatment of urinary tract bacterial infections. Nanotechnology. 2015;26:495602.

172. Nagavarma BVN, Yadav HKS, Ayaz A, Vasudha LS, Shivakumar HG. Different techniques for preparation of polymeric nanoparticles-a review. Asian J Pharm Clin Res. 2012;5(3):16-23.

173. Wischke C, Schwendeman SP. Principles of encapsulating hydrophobic drugs in PLA / PLGA microparticles. Int J Pharm. 2008;364:298-327.

174. Dhas N, Mehta T. Cationic biopolymer functionalized nanoparticles encapsulating lutein to attenuate oxidative stress in effective treatment of Alzheimer's disease: a non-invasive approach. Int J Pharm. 2020:586:119553.

175. Jia Y-P, Sun L, Yu H-S, Liang L-P, Li W, Ding H, et al. The Pharmacological effects of lutein and zeaxanthin on visual disorders and cognition diseases. Molecules. 2017;22(4):610.

176. Sathya S, Shanmuganathan B, Balasubramaniam B, Balamurugan K Devi KP. Phytol loaded PLGA nanoparticles regulate the expression of Alzheimer's related genes and neuronal apoptosis against amyloid- $\beta$ induced toxicity in Neuro-2a cells and transgenic Caenorhabditis elegans. Food Chem Toxicol. 2020;136:110962.

177. Islam MT, Ali ES, Uddin SJ, Shaw S, Islam MA, Ahmed MI, et al. Phytol: a review of biomedical activities. Food Chem Toxicol. 2019;121:82-94. 
178. Cano A, Ettcheto M, Chang J, Barroso E, Espina M, Kühne B, et al. Dual-drug loaded nanoparticles of Epigallocatechin-3-gallate ( EGCG )/ Ascorbic acid enhance therapeutic efficacy of EGCG in a APPswe / PS1dE9 Alzheimer's disease mice model. J Control Release. 2019;301:62-75.

179. Chowdhury A, Sarkar J, Chakraborti T, Pramanik PK, Chakraborti S. Protective role of epigallocatechin-3-gallate in health and disease: a perspective. Biomed Pharmacother. 2016;78:50-9.

180. Ahlschwede KM, Curran GL, Rosenberg JT, Grant SC, Sarkar G, Jenkins $\mathrm{RB}$, et al. Cationic carrier peptide enhances cerebrovascular targeting of nanoparticles in Alzheimer's disease brain. Nanomed Nanotechnol Biol Med. 2019;16:258-66.

181. Liu C, Lu D, You X, Shi G, Deng J. Carbon dots sensitized lanthanide infinite coordination polymer nanoparticles: towards ratiometric fluorescent sensing of cerebrospinal $A \beta$ monomer as a biomarker for Alzheimer's disease. Anal Chim Acta. 2020;1 105:147-54.

182. Jeon SG, Cha M, Kim J, Hwang TW, Kim KA, Kim TH, et al. Vitamin D-binding protein-loaded PLGA nanoparticles suppress Alzheimer's disease-related pathology in 5XFAD mice. Nanomed Nanotechnol Biol Med. 2019:17:297-307.

183. Zhang J, Sokal I, Peskind ER, Quinn JF, Jankovic J, Kenney C, et al. CSF multianalyte profile distinguishes Alzheimer and Parkinson diseases. Am J Clin Pathol. 2008;129(4):526-9.

184. Bishnoi RJ, Palmer RF, Royall DR. Vitamin D binding protein as a serum biomarker of Alzheimer's disease. J Alzheimers Dis. 2015;43(1):37-45.

185. Guo Q, Xu S, Yang P, Wang P, Lu S, Sheng D, et al. A dual-ligand fusion peptide improves the brain-neuron targeting of nanocarriers in Alzheimer's disease mice. J Control Release. 2020;320:347-62.

186. Klebowski B, Depciuch J, Parlinska-Wojtan M, Baran J. Applications of noble metal-based nanoparticles in medicine. Int J Mol Sci. 2018:19:4031.

187. Sánchez-lópez E, Gomes D, Esteruelas G, Bonilla L, Lopez-machado AL, Galindo R, et al. Metal-based nanoparticles as antimicrobial agents: an overview. Nanomaterials. 2020;10(2):292.

188. Khan ZUH, Khan A, Chen Y, Shah NS, Muhammad N, Khan AU, et al. Biomedical applications of green synthesized Nobel metal nanoparticles. J Photochem Photobiol B. 2017;173:150-64.

189. Govindrao P, Ghule NW, Haque A, Kalaskar MG. Metal nanoparticles synthesis: an overview on methods of preparation, advantages and disadvantages, and applications. J Drug Deliv Sci Technol. 2019;53:101174.

190. Rana A, Yadav K, Jagadevan S. A comprehensive review on green synthesis of nature-inspired metal nanoparticles: Mechanism, application and toxicity. J Cleaner Prod. 2020;122880.

191. Wu K, Su D, Liu J, Saha R, Wang J-P. Magnetic nanoparticles in nanomedicine: a review of recent advances. Nanotechnology. 2019;30(50):502003.

192. da Silva W, Brett CMA. Novel biosensor for acetylcholine based on acetylcholinesterase / poly (neutral red) — deep eutectic solvent / Fe2O3 nanoparticle modified electrode. J Electroanal Chem. 2020;872:114050.

193. Xing Y, Feng X-Z, Zhang L, Hou J, Han G-C, Chen Z. A sensitive and selective electrochemical biosensor for the determination of beta-amyloid oligomer by inhibiting the peptide-triggered in situ assembly of silver nanoparticles. Int J Nanomed. 2017;12:3171-9.

194. Liu Y, Zhou H, Yin T, Gong Y, Yuan G, Chen L, et al. Quercetin-modified gold-palladium nanoparticles as a potential autophagy inducer for the treatment of Alzheimer's disease. J Colloid Interf Sci. 2019;552:388-400.

195. Gao F, Zhao J, Liu P, Ji D, Zhang L, Zhang M, et al. Preparation and in vitro evaluation of multi-target-directed selenium- chondroitin sulfate nanoparticles in protecting against the Alzheimer 's disease. Int J Biol Macromol. 2020;142:265-76.

196. Kwok JCF, Warren P, Fawcett JW. Chondroitin sulfate: a key molecule in the brain matrix. Int J Biochem Cell Biol. 2012;44(4):582-6.

197. Solovyev ND. Importance of selenium and selenoprotein for brain function: from antioxidant protection to neuronal signalling. J Inorg Biochem. 2015;153:1-12.

198. Sonawane SK, Ahmad A, Chinnathambi S. Protein-capped metal nanoparticles inhibit tau aggregation in Alzheimer's Disease. ACS Omega. 2019;4:12833-40.

199. Yang L, Yin T, Liu Y, Sun J, Zhou Y, Liu J. Gold nanoparticle-capped mesoporous silica-based $\mathrm{H} 2 \mathrm{O} 2$-responsive controlled release system for Alzheimer's disease treatment. Acta Biomater. 2016;46:177-90.

200. Zhang L, Zhao P, Yue C, Jin Z, Liu Q, Du X, et al. Sustained release of bioactive hydrogen by Pd hydride nanoparticles overcomes Alzheimer's disease. Biomaterials. 2019;197:393-404.

201. Cai J, Dao P, Chen H, Yan L, Liang Y, Zhang W, et al. Ultrasmall superparamagnetic iron oxide nanoparticles-bound NIR dyes: novel theranostic agents for Alzheimer's disease. Dyes Pigm. 2020;173:107968.

202. Wahle T, Sofranko A, Dekkers S, Miller MR, Heusinkveld HJ, Albrecht C, et al. Evaluation of neurological effects of cerium dioxide nanoparticles doped with different amounts of zirconium following inhalation exposure in mouse models of Alzheimer 's and vascular disease. Neurochem Int. 2020;138:104755.

\section{Publisher's Note}

Springer Nature remains neutral with regard to jurisdictional claims in published maps and institutional affiliations.

Ready to submit your research? Choose BMC and benefit from:

- fast, convenient online submission

- thorough peer review by experienced researchers in your field

- rapid publication on acceptance

- support for research data, including large and complex data types

- gold Open Access which fosters wider collaboration and increased citations

- maximum visibility for your research: over 100M website views per year

At BMC, research is always in progress.

Learn more biomedcentral.com/submissions 